TRANSACTIONS OF THE

AMERICAN MATHEMATICAL SOCIETY

Volume 358, Number 3, Pages 1033-1049

S 0002-9947(05)03700-1

Article electronically published on April 13, 2005

\title{
THE INTEGRAL COHOMOLOGY OF THE BIANCHI GROUPS
}

\author{
ETHAN BERKOVE
}

\begin{abstract}
We calculate the integral cohomology ring structure for various members of the Bianchi group family. The main tools we use are the Bockstein spectral sequence and a long exact sequence derived from Bass-Serre theory.
\end{abstract}

\section{IntRoduCtion}

In order to determine $H^{*}(G ; M)$, the cohomology of a group $G$ with coefficients in $M$, it is standard practice to let $M$ be a field in order to simplify the calculations. The integral cohomology ring, with $M=\mathbb{Z}$, can be much harder to determine, particularly when the group contains a lot of torsion. However, in many cases it is still possible to get a complete answer. In this paper we calculate the integral cohomology rings for a number of Bianchi groups.

The Bianchi groups are a family of matrix groups whose entries are elements in a ring of algebraic integers. The Bianchi groups are of interest as they appear in numerous contexts, including the study of knots and 3 manifolds, and in the theory of automorphic forms (see [7] for references). The Bianchi groups are particularly amenable to calculation as their torsion occurs only at the primes 2 and 3 , their mod-2 cohomology rings and integral homology groups are known, and their 3 -primary cohomology is easily calculable. The groups themselves can be built in stages out of finite groups using amalgamated products and HNN extensions. Therefore, their group cohomology is closely related to the cohomology of these finite pieces.

This paper completes a calculation that has partially appeared in a number of places: For various members of the Bianchi group family, the integral homology (additive structure) can be found in [13, the rational homology in [16], and the mod-2 cohomology in [2]. The main tools we use at the prime 2 are the Bockstein spectral sequence and long exact sequences that come from Bass-Serre theory. At the prime 3 we perform the calculations solely using long exact sequences. Our calculations show, among other things, that despite a fairly complicated ring structure, $H^{*}\left(\Gamma_{5} ; \mathbb{Z}\right)$ and $H^{*}\left(\Gamma_{10} ; \mathbb{Z}\right)$ only differ by a few torsion-free classes. Given the difference in structure between $\Gamma_{5}$ and $\Gamma_{10}$, we find this similarity striking.

We have organized this paper as follows. In the next section we describe the Bianchi groups and the techniques we will need to determine their integral cohomology rings. In the third section we apply these techniques to determine some

Received by the editors November 13, 2002 and, in revised form, April 1, 2004.

2000 Mathematics Subject Classification. Primary 20J06, 11F75, 22E40.

Key words and phrases. Integral cohomology, Bianchi group, Bass-Serre theory.

(C)2005 American Mathematical Society Reverts to public domain 28 years from publication 1033 
intermediate results. In the fourth section we give complete details for the calculation of $H^{*}(G ; \mathbb{Z})$, where $G=\Gamma_{2}, \Gamma_{6}$. In the final section we do the cases $G=\Gamma_{1}, \Gamma_{3}, \Gamma_{5}, \Gamma_{7}, \Gamma_{10}, \Gamma_{11}$.

\section{BACKGROUND}

For $d$ a square-free integer, let $\mathcal{O}_{d}$ denote the ring of algebraic integers in the imaginary quadratic extension field of the rationals, $\mathbb{Q}(\sqrt{-d})$. As a $\mathbb{Z}$-module, $\mathcal{O}_{d} \cong \mathbb{Z} \oplus \mathbb{Z} \omega$, where $\omega=\sqrt{-d}$ when $d \equiv 3 \bmod 4$ and $\omega=\frac{1+\sqrt{-d}}{2}$ when $d \equiv 1,2 \bmod 4$. The Bianchi groups $\Gamma_{d}$ are the projective special linear groups $P S L_{2}\left(\mathcal{O}_{d}\right)$, and can be thought of as the natural generalizations of the modular group, $P S L_{2}(\mathbb{Z}) \cong \mathbb{Z} / 2 * \mathbb{Z} / 3$.

The Bianchi groups are excellent candidates for integral cohomology calculations. Torsion in Bianchi groups occurs at the primes 2 and 3, so one only needs to perform two $p$-primary calculations. Furthermore, the most complicated torsion occurs at the prime $p=2$, and mod-2 cohomology rings (as modules over the Steenrod algebra) have already been calculated for the Bianchi groups with $d=$ $1,2,3,5,6,7,10$ and 11 in [2].

Starting with mod- $p$ cohomology information, the $p$-primary component of the integral cohomology can be reconstructed using a handy tool: the Bockstein spectral sequence. This spectral sequence, which first appeared in [3], is associated to the following exact couple:

$$
\ldots \stackrel{\delta}{\longrightarrow} H^{*}(X ; \mathbb{Z}) \stackrel{\left(\times p^{*}\right)}{\longrightarrow} H^{*}(X ; \mathbb{Z}) \stackrel{r e d_{p^{*}}}{\longrightarrow} H^{*}\left(X ; \mathbb{F}_{p}\right) \stackrel{\delta}{\longrightarrow} H^{*+1}(X ; \mathbb{Z}) \stackrel{\left(\times p^{*}\right)}{\longrightarrow} \ldots
$$

The first differential is $d_{1}=\operatorname{red}_{p^{*}} \circ \delta$. In fact $d_{1}=\beta$, the classical Bockstein homomorphism, which can be identified with the Steenrod square $S q^{1}$ when $p=2$. Therefore, if $S q^{1}(w) \neq 0$ for some class $w \in H^{n}\left(X ; \mathbb{F}_{2}\right)$, then $\delta(w)$ represents some cohomology class $\hat{w} \in H^{n+1}(X ; \mathbb{Z})$ of order 2 . Elements that appear in the integral cohomology at different pages of the spectral sequence have different orders; the $r^{t h}$ differential $d_{r}$ can be identified with the $r^{\text {th }}$ order Bockstein homomorphism [9]

$$
\beta_{r}: H^{*}\left(X ; \mathbb{Z} / p^{r} \mathbb{Z}\right) \stackrel{\times p^{r-1}}{\longrightarrow} H^{*+1}\left(X ; \mathbb{Z} / p^{r} \mathbb{Z}\right) .
$$

Thus, elements that survive to the $E_{r}$ page have order at least $p^{r}$. The Bockstein spectral sequence converges to $\left(H^{*}(X ; \mathbb{Z}) / p\right.$-torsion $) \otimes_{\mathbb{Z}} \mathbb{F}_{p}$. We use this sequence to calculate group cohomology via the identification $H^{*}(G ; M)=H^{*}(B G ; M)$, where $B G$ is the classifying space of $G$.

For the Bianchi groups we consider, the Bockstein spectral sequence applied to the calculations in 2 almost collapses at the $E_{1}$ page, with at most one torsion class surviving to the $E_{2}$ page. Thus, almost all 2-torsion in the resulting integral cohomology rings has order 2, a result consistent with the calculations in [13. Although it would be convenient if this were the only tool needed in this paper, the Bockstein spectral sequence is less effective at identifying torsion-free classes, where knowledge of all Bockstein homomorphisms is required. Further, we need a technique to determine the 3-primary cohomology ring.

Fortunately, there is another technique. The Bianchi groups we consider in this article are built in stages using amalgamated products and HNN extensions from copies of $\mathbb{Z}$ and five finite subgroups: the cyclic groups of order two and three $(\mathbb{Z} / 2$ and $\mathbb{Z} / 3)$, the Klein four group $\left(\mathbf{D}_{2} \cong \mathbb{Z} / 2 \times \mathbb{Z} / 2\right)$, the symmetric group on three letters $\left(\mathbf{S}_{3}\right)$, and the alternating group on four letters $\left(\mathbf{A}_{4}\right)$ [7. We show how the 
cohomology of a group built via these constructions depends on the cohomology of its component subgroups. This then allows us to determine the torsion-free classes as well as the 3-primary cohomology ring. At the prime 2, this approach is also helpful for identifying the few classes of order 4 in the 2-primary cohomology ring.

We summarize some useful results, starting with the definitions of the amalgamated product and the HNN extension. A more thorough treatment can be found in $[2$.

Definition 2.1. Let $G_{1}$ and $G_{2}$ be groups with a common subgroup $H$. Let $\theta_{i}: H \rightarrow G_{i}$ be monomorphisms. The amalgamated product $G_{1} *_{H} G_{2}$ is a group

$$
\Gamma=\left\{G_{1}, G_{2} \mid \theta_{1}(h)=\theta_{2}(h), h \in H\right\} .
$$

Similarly, an $H N N$ extension of $G_{1}$ is a group

$$
\Gamma=\left\{t, G_{1} \mid t^{-1} h t=\theta_{1}(h), h \in H\right\} .
$$

$\Gamma$ is denoted by $G_{1} *_{H} . G_{1}$ is called the base and $H$ is called the associated subgroup.

When $\Gamma \cong G_{1} *_{H} G_{2}$ is an amalgamated product or $\Gamma \cong G_{1} *_{H}$ is an HNN extension, Bass-Serre theory (14, page 127) provides a long exact sequence with coefficients in $M$ :

$$
\ldots \stackrel{\alpha}{\longrightarrow} H^{j-1}(H ; M) \stackrel{\delta}{\longrightarrow} H^{j}(\Gamma ; M) \stackrel{\rho}{\longrightarrow} \oplus H^{j}\left(G_{i} ; M\right) \stackrel{\alpha}{\longrightarrow} H^{j}(H ; M) \stackrel{\delta}{\longrightarrow} \ldots
$$

The maps above can be explicitly described. The map $\rho$ is a sum of restriction maps to each summand. When $\Gamma$ is an amalgamated product, $\alpha$ is the difference of restriction maps, $\alpha=\operatorname{res}_{H}^{G_{1}}-\operatorname{res}_{H}^{G_{2}}$. When $G$ is an HNN extension, the "twisting" induced by $\theta$ comes into play, and $\alpha=\operatorname{res}_{H}^{G_{1}}-\theta^{*}$.

Remark 2.2. Whenever $\Gamma$ is an HNN extension, $\rho$ is an isomorphism in degree zero. Consequently, $\alpha$ factors through zero and $\delta$ is a monomorphism, generating a class in degree 1 . We call such classes "HNN classes". We note that with integral coefficients, HNN classes are torsion-free, and cup trivially with themselves as they have odd degree. Therefore, they are always exterior.

The map $\rho$ respects cup products, since it is a restriction map in the case of an HNN extension and a sum of restrictions in the case of an amalgamated product. Thus, by exactness of $H^{*}(\Gamma ; M) \stackrel{\rho}{\longrightarrow} \oplus H^{*}\left(G_{i} ; M\right) \stackrel{\alpha}{\longrightarrow} H^{*}(H ; M)$, we have that $i m(\rho) \cong k e r(\alpha)$, and this is an isomorphism of rings. In most of our calculations the map $\alpha$ is surjective, so sequence (2.1) breaks into short exact sequences. Hence the ring isomorphism determines most of the cup product structure of $H^{*}(\Gamma ; M)$, which can be used to determine cup products in the cases where the Bockstein spectral sequence information is incomplete or ambiguous.

In those cases where $\alpha$ is not surjective, classes can also arise in $H^{*}(\Gamma ; M)$ from the image of $\delta$. The following lemmas describe how to determine products with these classes.

Lemma 2.3 (5.6.12 in [15]). For $u \in H^{p}(\Gamma ; M)$ and $v \in H^{q}(H ; M), \delta\left(\operatorname{res}_{H}^{\Gamma_{H}} u \cup v\right)=$ $u \cup \delta(v)$, and $\delta\left(v \cup r e s_{H}^{\Gamma} u\right)=\delta(v) \cup u$.

Lemma 2.4. Classes that arise from the image of $\delta$ have trivial products with each other.

Proof. See Proposition 5.3 in [12]. 
Finally, on occasion we will need to determine restriction maps on integral cohomology. If the restriction map is known at the level of mod-2 cohomology, one can extend the map by appealing to the following long exact sequences of commuting squares:

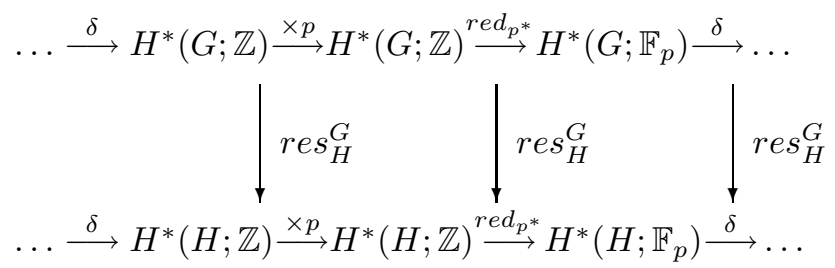

\section{INITIAL CALCULATIONS}

We determine the integral cohomology of the five finite groups that form the basis of the Bianchi groups' cohomology, then apply techniques from Section 2 to determine some intermediate results. In what follows, the subscript on a cohomology class denotes the degree of the class. A class inside square brackets is polynomial, whereas a class inside of parentheses is exterior.

Lemma 3.1. $H^{*}(\mathbb{Z} / 2 ; \mathbb{Z}) \cong \mathbb{Z}\left[x_{2}\right]$ where $x_{2}$ has order 2 . $H^{*}(\mathbb{Z} / 3 ; \mathbb{Z}) \cong \mathbb{Z}\left[x_{2}\right]$ where $x_{2}$ has order 3 .

Lemma 3.2. The 2-primary component $H^{*}\left(\mathbf{S}_{3} ; \mathbb{Z}\right)_{(2)} \cong H^{*}(\mathbb{Z} / 2 ; \mathbb{Z}) \cong \mathbb{Z}\left[x_{2}\right]$, where $x_{2}$ has order 2 , and the isomorphism is induced by restriction. The 3-primary component $H^{*}\left(\mathbf{S}_{3} ; \mathbb{Z}\right)_{(3)} \cong \mathbb{Z}\left[x_{4}\right]$ where $x_{4}$ has order 3 , and this is the image of $\operatorname{res}_{\mathbb{Z} / 3}^{\mathbf{S}_{3}}$.

Proof. Consider the short exact sequence

$$
0 \longrightarrow \mathbb{Z} / 3 \longrightarrow \mathbf{S}_{3} \longrightarrow \mathbb{Z} / 2 \longrightarrow 0 \text {. }
$$

There is a spectral sequence with $E_{2}^{p, q} \cong H^{p}\left(\mathbb{Z} / 2 ; H^{q}(\mathbb{Z} / 3 ; \mathbb{Z})\right)$ converging to $H^{*}\left(\mathbf{S}_{3} ; \mathbb{Z}\right)$. The horizontal edge of the spectral sequence is isomorphic to $H^{*}(\mathbb{Z} / 2 ; \mathbb{Z})$. The vertical edge, however, is the invariants $H^{*}(\mathbb{Z} / 3 ; \mathbb{Z})^{\mathbb{Z} / 2}$. The horizontal edge yields the 2-primary part of the cohomology. On the vertical edge, the action is free in dimensions $4 n+2$ and trivial otherwise (see [4], V.3, Exercise 4).

A calculation of the integral cohomology of $\mathbf{D}_{2}$ can be found in the literature.

Lemma 3.3 ([8], Proposition 4.1$) \cdot H^{*}\left(\mathbf{D}_{2} ; \mathbb{Z}\right) \cong \mathbb{Z}\left[y_{2}, z_{2}, y_{3}\right]$ with relation $y_{3}^{2}=$ $y_{2}^{2} z_{2}+y_{2} z_{2}^{2}$. All classes have order 2 .

The integral cohomology of $\mathbf{A}_{4}$ is harder to determine. We supply a calculation here using the Bockstein spectral sequence.

Lemma 3.4. $H^{*}\left(\mathbf{A}_{4} ; \mathbb{Z}\right)_{(2)} \cong \mathbb{Z}\left[y_{3}, y_{4}, y_{6}\right]$ with relation $y_{3}^{4}+y_{4}^{3}+y_{3}^{2} y_{6}+y_{6}^{2}=0$. All classes have order 2 . $H^{*}\left(\mathbf{A}_{4} ; \mathbb{Z}\right)_{(3)} \cong H^{*}(\mathbb{Z} / 3 ; \mathbb{Z})$, and this isomorphism is given by $\operatorname{res}_{\mathbb{Z} / 3}^{\mathbf{A}_{4}}$.

Proof. At the prime 2, start with $H^{*}\left(\mathbf{A}_{4} ; \mathbb{F}_{2}\right) \cong \mathbb{F}_{2}\left[u_{2}, v_{3}, w_{3}\right]$ with relation $u_{2}^{3}+$ $v_{3}^{2}+w_{3}^{2}+v_{3} w_{3}=0$ (see [1] for a derivation). The Bocksteins are $S q^{1}\left(u_{2}\right)=v_{3}$, $S q^{1}\left(v_{3}\right)=0$, and $S q^{1}\left(w_{3}\right)=u_{2}^{2}$. We identify three classes in $H^{*}\left(\mathbf{A}_{4} ; \mathbb{Z}\right)_{(2)}$ in low 
degrees: $y_{3}$, whose image under $\operatorname{red}_{2^{*}}$ is $v_{3}=S q^{1}\left(u_{2}\right) ; y_{4}$, whose image under $\operatorname{red}_{2^{*}}$ is $u_{2}^{2}=S q^{1}\left(w_{3}\right)$; and $y_{6}$, whose image under $\operatorname{red}_{2^{*}}$ is $u_{2}^{3}+v_{3} w_{3}=S q^{1}\left(u_{2} w_{3}\right)$. $S q^{1}\left(u_{2} v_{3}\right)=v_{3}^{2}$ is also a non-trivial square, but this class is the image of $y_{3}^{2}$ under red $_{2^{*}}$ and has thus already been accounted for. In what follows, we denote these identifications using a double arrow: $y_{3} \leftrightarrow v_{3}, y_{4} \leftrightarrow u_{2}^{2}$, and $y_{6} \leftrightarrow u_{2}^{3}+v_{3} w_{3}$.

One easily confirms that the classes $v_{3}, u_{2}^{2}, v_{3}^{2}$, and $u_{2}^{3}+v_{3} w_{3}$ generate the kernel of $S q^{1}$ up to degree 6 ; therefore, $y_{3}, y_{4}$, and $y_{6}$ are of order 2. A Poincaré series argument derived from the Universal Coefficient Theorem implies that these classes satisfy a single relation in degree 12. Explicitly

$$
\begin{aligned}
\left(y_{3}^{4}+y_{4}^{3}+y_{3}^{2} y_{6}+y_{6}^{2}\right) & \leftrightarrow v_{3}^{4}+u_{2}^{6}+v_{3}^{2}\left(u_{2}^{3}+v_{3} w_{3}\right)+u_{2}^{6}+v_{3}^{2} w_{3}^{2} \\
& \leftrightarrow v_{3}^{2}\left(u_{2}^{3}+v_{3}^{2}+v_{3} w_{3}+w_{3}^{2}\right)=0 .
\end{aligned}
$$

The last equality follows as $u_{2}^{3}+v_{3}^{2}+w_{3}^{2}+v_{3} w_{3}=0$ is a relation in $H^{*}\left(\mathbf{A}_{4} ; \mathbb{F}_{2}\right)$.

At the prime 3 , we note that $\mathbb{Z} / 3$ is self-normalizing in $\mathbf{A}_{4} . H^{*}\left(\mathbf{A}_{4} ; \mathbb{Z}\right)_{(3)}$ maps

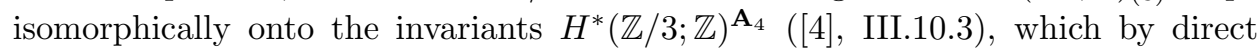
calculation is $H^{*}(\mathbb{Z} / 3 ; \mathbb{Z})$. The invariants are also the image of the restriction map.

Most of the restriction maps we will need are straightforward. However, $r e s_{\mathbb{Z} / 2}^{\mathbf{A}_{4}}$ is somewhat more complicated.

Lemma 3.5. Let $y_{3}, y_{4}$, and $y_{6}$ be the generators of $H^{*}\left(\mathbf{A}_{4} ; \mathbb{Z}\right)_{(2)}$. The map res $\mathbf{A}_{\mathbb{Z} / 2}^{\mathbf{A}_{4}}$ on integral cohomology sends $y_{4}^{i} y_{6}^{j}$ to $x_{2}^{2 i+3 j}$ and all other classes to 0 , where $x_{2}$ generates $H^{*}(\mathbb{Z} / 2 ; \mathbb{Z})$.

Proof. We use the commutative square:

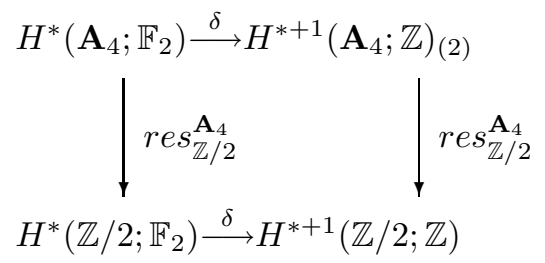

Let $u_{2}, v_{3}$, and $w_{3}$ generate $H^{*}\left(\mathbf{A}_{4} ; \mathbb{F}_{2}\right)$, and let $x_{1}$ and $x_{2}$ generate $H^{1}\left(\mathbb{Z} / 2 ; \mathbb{F}_{2}\right)$ and $H^{2}(\mathbb{Z} / 2 ; \mathbb{Z})$, respectively. The map $\operatorname{res}_{\mathbb{Z} / 2}^{\mathbf{A}_{4}}$ on the left sends $u_{2}$ to $x_{1}^{2}, v_{3}$ to 0 , and $w_{3}$ to $x_{1}^{3}$ (see Lemma 2.2 of [2]). By extension, $u_{2}^{i} w_{3}^{j}$ is mapped to $x_{1}^{2 i+3 j}$. The bottom map sends $x_{1}^{2 i-1}$ to $x_{2}^{i}$, so the composition is non-zero only when $j$ is odd. Since we determined the top map in Lemma 3.4, we should be able to determine the restriction map on the right.

Consider the class $u_{2}^{2 i+1} w_{3}^{2 j-1}$ that maps non-trivially to $x_{2}^{2 i+3 j}$ under the composition of the left and bottom maps. $S q^{1}\left(u_{2}^{2 i+1} w_{3}^{2 j-1}\right)=u_{2}^{2 i} w_{3}^{2 j-2}\left(u_{2}^{3}+v_{3} w_{3}\right)$, which corresponds to the class $y_{4}^{i}\left(y_{3}^{2}+y_{6}\right)^{j-1} y_{6}$ in the integral cohomology. Similarly, $u_{2}^{2 i} w_{3}^{2 j-1}$ corresponds to the class $y_{4}^{i+1}\left(y_{3}^{2}+y_{6}\right)^{j-1}$. We claim that the only components in the expansion of these products with non-trivial images under $r e s_{\mathbb{Z} / 2}^{\mathbf{A}_{4}}$ are the terms $y_{4}^{i} y_{6}^{j}$.

Note that if $S q^{1}(z)=0$, then $S q^{1}(w z)=\left(S q^{1} w\right) z$ for any class $w$ by the Cartan formula. In $H^{*}\left(\mathbf{A}_{4} ; \mathbb{F}_{2}\right), u_{2}^{3}+v_{3} w_{3}=v_{3}^{2}+w_{3}^{2}$. Thus, $S q^{1}\left(u_{2}^{3}+v_{3} w_{3}\right)=0$, so $S q^{1}\left(u_{2}^{2 j+1} v_{3}^{i-1}\left(u_{2}^{3}+v_{3} w_{3}\right)^{k}\right)=u_{2}^{2 j} v_{3}^{i}\left(u_{2}^{3}+v_{3} w_{3}\right)^{k}$, which corresponds to the class 
$y_{3}^{i} y_{4}^{j} y_{6}^{k}$. On the other hand, since any term in $H^{*}\left(\mathbf{A}_{4} ; \mathbb{F}_{2}\right)$ containing a $v_{3}$ maps to zero under $\mathrm{res}_{\mathbb{Z} / 2}^{\mathbf{A}_{4}}, y_{3}^{i} y_{4}^{j} y_{6}^{k}$ must map to 0 also.

To finish the argument, $S q^{1}\left(u_{2}^{2 i-2} w_{3}\right)$ corresponds to $y_{4}^{i}$ and maps non-trivially onto $x_{2}^{2 i}$. Therefore, the restriction map is only non-zero when it sends $y_{4}^{i} y_{6}^{j}$ to $x_{2}^{2 i+3 j}$.

Remark 3.6. Using a similar but easier analysis, one can show that $r e s_{\mathbb{Z} / 2}^{\mathbf{D}_{2}}$ sends powers of $x_{2} \in H^{2}\left(\mathbf{D}_{2} ; \mathbb{Z}\right)$ isomorphically to powers of the corresponding generator of $H^{2}(\mathbb{Z} / 2 ; \mathbb{Z})$ and vanishes otherwise.

We next move to the calculation of three amalgamated products that appear as base groups for a number of the Bianchi groups presented in this article. In many cases, a Bianchi group's cohomology is nearly identical to its base group's, so the following results consolidate later calculations.

Lemma 3.7. $H^{*}\left(\mathbf{D}_{2} *_{\mathbb{Z} / 2} \mathbf{D}_{2} ; \mathbb{Z}\right) \cong \mathbb{Z}\left[x_{2}, y_{2}, z_{2}, y_{3}, z_{3}\right]$ with relations $y_{2} z_{3}=z_{2} y_{3}=$ $0, y_{3}^{2}=x_{2} y_{2}\left(x_{2}+y_{2}\right)$, and $z_{3}^{2}=x_{2} z_{2}\left(x_{2}+z_{2}\right)$. All classes have order 2 .

Proof. We use sequence (2.1) with integer coefficients:

$$
\stackrel{\delta}{\longrightarrow} H^{*}\left(\mathbf{D}_{2} * \mathbb{Z} / 2 \mathbf{D}_{2} ; \mathbb{Z}\right) \stackrel{\rho}{\longrightarrow} H^{*}\left(\mathbf{D}_{2} ; \mathbb{Z}\right) \oplus H^{*}\left(\mathbf{D}_{2} ; \mathbb{Z}\right) \stackrel{\alpha}{\longrightarrow} H^{*}(\mathbb{Z} / 2 ; \mathbb{Z}) \stackrel{\delta}{\longrightarrow} .
$$

By Remark 3.6 $\alpha$ is surjective, so this sequence breaks into short exact sequences. Let $x_{2}, y_{2}, y_{3}$ and $x_{2}^{\prime}, z_{2}, z_{3}$ be generators in each copy of $H^{*}\left(\mathbf{D}_{2} ; \mathbb{Z}\right)$ in the direct sum, and let $w_{2}$ be the generator of $H^{2}(\mathbb{Z} / 2)$ that is the restriction of both $x_{2}$ and $x_{2}^{\prime}$ under $\alpha$. Now $y_{2}, z_{2}, y_{3}, z_{3}$ and $x_{2}+x_{2}^{\prime}$ are all in $k e r(\alpha)$ and have order 2. Since this kernel is isomorphic to the image of $\rho$, we can construct the cohomology ring structure of $\mathbf{D}_{2} * \mathbb{Z} / 2 \mathbf{D}_{2}$ at this point. We abuse notation and refer to classes in $H^{*}\left(\mathbf{D}_{2} * \mathbb{Z} / 2 \mathbf{D}_{2} ; \mathbb{Z}\right)$ by their image under $\rho$, although we rename $x_{2}+x_{2}^{\prime}$ as $\bar{x}_{2}$. Most of the relations are obvious; note that $y_{3}^{2}=\bar{x}_{2} y_{2}\left(\bar{x}_{2}+y_{2}\right)$ as products of $x_{2}^{\prime}$ with $y_{2}$ equal 0 . That the relations we found form a minimal set follows from a Poincaré series argument. We drop the bar on the $x_{2}$ for brevity.

One can derive the same result by using the Bockstein spectral sequence. Briefly, start with $H^{*}\left(\mathbf{D}_{2} *_{\mathbb{Z} / 2} \mathbf{D}_{2} ; \mathbb{F}_{2}\right) \cong \mathbb{F}_{2}\left[p_{1}, q_{1}, r_{1}\right] / p_{1} q_{1}=0$ [2] and make the following identifications: $x_{2} \leftrightarrow r_{1}^{2}, y_{2} \leftrightarrow p_{1}^{2}, z_{2} \leftrightarrow q_{1}^{2}, y_{3} \leftrightarrow p_{1} r_{1}\left(p_{1}+r_{1}\right)$, and $z_{3} \leftrightarrow q_{1} r_{1}\left(q_{1}+r_{1}\right)$. These are the Bocksteins of the classes $r_{1}, p_{1}, q_{1}, p_{1} r_{1}$, and $q_{1} r_{1}$, respectively. It is straightforward to confirm that the stated relations hold.

For $\mathbf{D}_{2} *_{\mathbb{Z} / 2} \mathbf{D}_{2}$, the integral calculations are possible using either the Bockstein spectral sequence or sequence (2.1). In practice, both techniques are often needed to determine the answer. The long exact sequence is an effective means to determine generators in low degree, and show exactly how classes arise from pieces of the amalgamated product. The Bockstein spectral sequence, on the other hand, is helpful in the determination of the relations between generators.

Lemma 3.8. $H^{*}\left(\mathbf{A}_{4} * \mathbb{Z} / 2 \mathbf{D}_{2} ; \mathbb{Z}\right)_{(2)} \cong \mathbb{Z}\left[y_{2}, y_{3}, z_{3}, y_{4}, z_{4}, y_{5}, y_{6}\right] /\langle R\rangle$. All classes have order two, and $\langle R\rangle$ is the set of relations given in Table 1 .

Proof. By results in [2], $H^{*}\left(\mathbf{A}_{4} *_{\mathbb{Z} / 2} \mathbf{D}_{2} ; \mathbb{F}_{2}\right) \cong \mathbb{F}_{2}\left[x_{1}, y_{2}, u_{2}, v_{3}, w_{3}\right]$ with relations $u_{2}^{3}+v_{3}^{2}+w_{3}^{2}+v_{3} w_{3}=0, x_{1}^{2} u_{2}=y_{2}^{2}, x_{1} w_{3}=u_{2} y_{2}, y_{2} w_{3}=x_{1} u_{2}^{2}$, and $x_{1} v_{3}=$ $y_{2} v_{3}=0$. In addition, $S q^{1}\left(x_{1}\right)=x_{1}^{2}, S q^{1}\left(y_{2}\right)=x_{1}\left(u_{2}+y_{2}\right), S q^{1}\left(u_{2}\right)=v_{3}$, and $S q^{1}\left(w_{3}\right)=u_{2}^{2}$. 
TABle 1. Relations by degree for $H^{*}\left(\mathbf{A}_{4} * \mathbb{Z} / 2 \mathbf{D}_{2} ; \mathbb{Z}\right)_{(2)}$

\begin{tabular}{|l|l|}
\hline Degree & Relation(s) \\
\hline 5 & $y_{2} z_{3}=0$ \\
\hline 6 & $y_{3} z_{3}=0, y_{2} y_{4}+y_{2} z_{4}+y_{3}^{2}=0$ \\
\hline 7 & $y_{2} y_{5}=y_{3} y_{4}, z_{3} y_{4}=0$ \\
\hline 8 & $y_{2} y_{6}+y_{3} y_{5}+y_{4}^{2}=0, y_{2} y_{6}=y_{4} z_{4}, y_{2}^{2} z_{4}=y_{4}^{2}, z_{3} y_{5}=0$ \\
\hline 9 & $y_{2} y_{3} z_{4}=y_{4} y_{5}, y_{3} y_{6}=z_{4} y_{5}$ \\
\hline 10 & $y_{2} z_{4}^{2}=y_{4} y_{6}, y_{3}^{2} z_{4}=y_{5}^{2}$ \\
\hline 11 & $y_{3} z_{4}^{2}=y_{5} y_{6}$ \\
\hline 12 & $y_{6}^{2}+y_{6} z_{3}^{2}+z_{3}^{4}+z_{4}^{3}=0$ \\
\hline
\end{tabular}

We apply the Bockstein spectral sequence and identify generators for the integral cohomology ring. In general, one writes down classes in the mod-2 cohomology by degree and determines their Steenrod squares. Every time a class in the integral cohomology ring is encountered that is not in the ideal generated by the previous classes, it is added to the generating set. Relations among classes of order 2 can be determined using the relations in the mod-2 cohomology ring as in Lemma 3.4 There are some ways to speed up this occasionally difficult process. Once all integral generators have been found, it is straightforward to write down all possible combinations of generators by degree. Using the Poincaré series for the mod-2 cohomology and the Universal Coefficient Theorem, one knows how many classes are in the integral cohomology in each degree; this determines the number of relations among the classes. One continues this process until an integral Poincaré series argument or other appropriate technique shows that all generators and relations have been found.

Following this process, we identify generators $y_{2} \leftrightarrow x_{1}^{2}=S q^{1}\left(x_{1}\right) ; y_{3} \leftrightarrow$ $x_{1}\left(u_{2}+y_{2}\right)=S q^{1}\left(y_{2}\right) ; z_{3} \leftrightarrow v_{3}=S q^{1}\left(u_{2}\right) ; y_{4} \leftrightarrow x_{1}^{2} u_{2}=S q^{1}\left(x_{1} u_{2}\right) ; z_{4} \leftrightarrow u_{2}^{2}=$ $S q^{1}\left(w_{3}\right) ; y_{5} \leftrightarrow x_{1}^{2} w_{3}+x_{1} u_{2}^{2}=S q^{1}\left(x_{1} w_{3}\right)$; and $y_{6} \leftrightarrow u_{2}^{3}+v_{3} w_{3}=S q^{1}\left(u_{2} w_{3}\right)$. As a sample confirmation of the relations, note that $y_{5}^{2} \leftrightarrow x_{1}^{2} u_{2}^{4}+x_{1}^{4} w_{3}^{2}$ and $y_{3} z_{4}^{2} \leftrightarrow x_{1}^{2} u_{2}^{4}+x_{1}^{2} u_{2}^{2} y_{2}^{2}$. Using the relation $x_{1} w_{3}=u_{2} y_{2}$ from the mod-2 ring, we find that the two expressions are equal. The other relations in Table 1 can be similarly checked.

In this example, the number of generators makes it difficult to confirm that the spectral sequence collapses at $E_{2}$. However, using sequence (2.1) we get

$$
\stackrel{\delta}{\longrightarrow} H^{*}\left(\mathbf{A}_{4} * \mathbb{Z} / 2 \mathbf{D}_{2} ; \mathbb{Z}\right)_{(2)} \stackrel{\rho}{\longrightarrow} H^{*}\left(\mathbf{A}_{4} ; \mathbb{Z}\right)_{(2)} \oplus H^{*}\left(\mathbf{D}_{2} ; \mathbb{Z}\right) \stackrel{\alpha}{\longrightarrow} H^{*}(\mathbb{Z} / 2 ; \mathbb{Z}) \stackrel{\delta}{\longrightarrow} .
$$

The map $\alpha$ is a surjection, which follows from Lemma 3.5 and Remark 3.6 Therefore, the long exact sequence breaks into short exact sequences and all 2-torsion in $H^{*}\left(\mathbf{A}_{4} * \mathbb{Z} / 2 \mathbf{D}_{2} ; \mathbb{Z}\right)_{(2)}$ must have order 2. This implies that $E_{2}=E_{\infty}$.

Lemma 3.9. $H^{*}\left(\mathbf{A}_{4} *_{\mathbb{Z} / 2} \mathbf{A}_{4} ; \mathbb{Z}\right)_{(2)} \cong \mathbb{Z}\left[y_{3}, y_{4}, y_{6}, z_{6}, y_{7}, y_{9}\right] /\langle R\rangle \widetilde{\oplus} \mathbb{Z}\left(t_{3}\right)$. The class $t_{3}$ has order 4 ; the other generators have order 2 . The set of relations, $\langle R\rangle$, is given in Table 2.

Proof. $H^{*}\left(\mathbf{A}_{4} * \mathbb{Z} / 2 \mathbf{A}_{4} ; \mathbb{F}_{2}\right) \cong \mathbb{F}_{2}\left[u_{2}, v_{3}, \bar{v}_{3}, w_{3}\right] \widetilde{\oplus} \mathbb{F}_{2}\left(s_{2}\right)$ with relations $u_{2}^{3}+w_{3}^{2}+$ $v_{3}^{2}+\bar{v}_{3}^{2}+w_{3}\left(v_{3}+\bar{v}_{3}\right)=0, v_{3} \bar{v}_{3}=0$ [2]. Also, $S q^{1}\left(u_{2}\right)=v_{3}+\bar{v}_{3}$ and $S q^{1}\left(w_{3}\right)=u_{2}^{2}$. 
TABle 2. Relations by degree for $H^{*}\left(\mathbf{A}_{4} * \mathbb{Z} / 2 \mathbf{A}_{4} ; \mathbb{Z}\right)_{(2)}$

\begin{tabular}{|l|l|}
\hline Degree & Relation(s) \\
\hline 10 & $y_{3} y_{7}=y_{4} z_{6}$ \\
\hline 12 & $y_{3}^{4}+y_{3}^{2} y_{6}+y_{4}^{3}+y_{6}^{2}=0, y_{3} y_{9}=y_{6} z_{6}, y_{3}^{2} z_{6}=z_{6}^{2}$ \\
\hline 13 & $y_{3}^{2} y_{7}=z_{6} y_{7}, y_{4} y_{9}=y_{6} y_{7}$ \\
\hline 14 & $y_{4}^{2} z_{6}=y_{7}^{2}$ \\
\hline 15 & $y_{3} y_{6} z_{6}=z_{6} y_{9}$ \\
\hline 16 & $y_{4} y_{6} z_{6}=y_{7} y_{9}$ \\
\hline 18 & $y_{3} y_{6} y_{9}=y_{9}^{2}, y_{3} z_{6} y_{9}+y_{4} y_{7}^{2}+y_{9}^{2}+z_{6}^{3}=0$ \\
\hline
\end{tabular}

We identify the following classes in the integral cohomology ring: $y_{3} \leftrightarrow v_{3}+\bar{v}_{3}=$ $S q^{1}\left(u_{2}\right) ; y_{4} \leftrightarrow u_{2}^{2}=S q^{1}\left(w_{3}\right) ; y_{6} \leftrightarrow u_{2}^{3}+\left(v_{3}+\bar{v}_{3}\right) w_{3}=S q^{1}\left(u_{2} w_{3}\right) ; z_{6} \leftrightarrow v_{3}^{2}=$ $S q^{1}\left(u_{2} v_{3}\right) ; y_{7} \leftrightarrow u_{2}^{2} v_{3}=S q^{1}\left(v_{3} w_{3}\right) ;$ and $y_{9} \leftrightarrow v_{3}\left(u_{2}^{3}+v_{3} w_{3}\right)=S q^{1}\left(u_{2} v_{3} w_{3}\right)$. An exhaustive search finds a set of relations among these classes.

Note that $S q^{1}\left(v_{3}\right)=S q^{1} \bar{v}_{3}=0$, whereas $S q^{1}\left(u_{2}\right)=v_{3}+\bar{v}_{3}$. This means that there is a class in the integral cohomology that has at least order 4 . We show that this class has exactly order 4 by considering the short exact sequence of 2-primary groups from sequence (2.1)

$0 \stackrel{\alpha}{\longrightarrow} H^{2}(\mathbb{Z} / 2 ; \mathbb{Z}) \stackrel{\delta}{\longrightarrow} H^{3}\left(\mathbf{A}_{4} *_{\mathbb{Z} / 2} \mathbf{A}_{4} ; \mathbb{Z}\right)_{(2)} \stackrel{\rho}{\longrightarrow} H^{3}\left(\mathbf{A}_{4} ; \mathbb{Z}\right)_{(2)} \oplus H^{3}\left(\mathbf{A}_{4} ; \mathbb{Z}\right)_{(2)} \stackrel{\alpha}{\longrightarrow} 0$.

Using Lemma 3.4, we substitute to get

$$
0 \longrightarrow \mathbb{Z} / 2 \longrightarrow H^{3}\left(\mathbf{A}_{4} *_{\mathbb{Z} / 2} \mathbf{A}_{4} ; \mathbb{Z}\right)_{(2)} \longrightarrow \mathbb{Z} / 2 \oplus \mathbb{Z} / 2 \longrightarrow 0
$$

We know from the Bockstein spectral sequence that there are two classes in $H^{3}\left(\mathbf{A}_{4} * \mathbb{Z} / 2 \mathbf{A}_{4} ; \mathbb{Z}\right)_{(2)}$, exactly one of which has order greater than two. This implies that $H^{3}\left(\mathbf{A}_{4} *_{\mathbb{Z} / 2} \mathbf{A}_{4} ; \mathbb{Z}\right)_{(2)} \cong \mathbb{Z} / 2 \oplus \mathbb{Z} / 4$. We denote the class of order 4 by $t_{3}$.

It remains to determine products with $t_{3}$. First, $t_{3}$ is exterior (that is, $t_{3}^{2}=0$ ), as $t_{3}$ is the only class in the cohomology ring of order 4 . Without loss of generality, we can say that $\rho\left(t_{3}\right)=v_{3}$. Since $\rho\left(y_{3}\right)=v_{3}+\bar{v}_{3}$, if $t_{3} y_{3}$ is a non-zero product it must be $z_{6}$, as both classes map to $v_{3}^{2}$ under $\rho$ and there is only one such class. But $z_{6}^{2} \neq 0$, whereas $\left(t_{3} y_{3}\right)^{2}=0$; we conclude that $t_{3} y_{3}=0$. Similar arguments show that all products with $t_{3}$ are 0 .

\section{The Bianchi groups $\Gamma_{2}$ And $\Gamma_{6}$}

In this section, we calculate the integral cohomology rings of $\Gamma_{2}$ and $\Gamma_{6}$ in detail. We use the Bockstein spectral sequence to determine the 2-torsion, and sequence (2.1) for the rest of the ring structure. In here and what follows, Roman letters refer to torsion classes, and Greek letters to torsion-free classes. A subscript denotes the degree of a class; classes in square brackets are polynomial, and classes in parentheses are exterior.

Theorem 4.1. $H^{*}\left(\Gamma_{2} ; \mathbb{Z}\right)_{(2)} \cong \mathbb{Z}\left[y_{2}, y_{3}, y_{4}, y_{5}, y_{6}, y_{7}\right]\left(t_{3}, \sigma_{1}\right) /\langle R\rangle$. The class $t_{3}$ has order four; the other torsion generators have order 2 . The set of relations, $\langle R\rangle$, is given in Table 3. $H^{*}\left(\Gamma_{2} ; \mathbb{Z}\right)_{(3)} \cong \mathbb{Z}\left[x_{2}\right]\left(\sigma_{1}\right)$ where $x_{2}$ has order 3 .

Proof. From [2], $H^{*}\left(\Gamma_{2} ; \mathbb{F}_{2}\right) \cong \mathbb{F}_{2}\left[n_{1}, m_{2}, n_{3}, m_{3}\right] \widetilde{\oplus} \mathbb{F}_{2}\left(s_{1}\right) \widetilde{\oplus} \mathbb{F}_{2}\left(s_{2}\right)$ with relations $n_{1} n_{3}=0$ and $m_{2}^{3}+m_{3}^{2}+n_{3}^{2}+m_{3} n_{3}+n_{1} m_{2} m_{3}=0$. All products with $\sigma_{1}$ vanish 
TABLE 3 . Relations by degree for $H^{*}\left(\Gamma_{2} ; \mathbb{Z}\right)_{(2)}$

\begin{tabular}{|l|l|}
\hline Degree & Relation(s) \\
\hline 3 & $\sigma_{1} y_{2}=2 t_{3}$ \\
\hline 4 & $\sigma_{1} t_{3}=\sigma_{1} y_{3}=0$ \\
\hline 5 & $\sigma_{1} y_{4}=y_{2} t_{3}=0$ \\
\hline 6 & $\sigma_{1} y_{5}=t_{3} y_{3}=0$ \\
\hline 7 & $\sigma_{1} y_{6}=t_{3} y_{4}=0$ \\
\hline 8 & $\sigma_{1} y_{7}=t_{3} y_{5}=0, y_{2}^{2} y_{4}=y_{2} y_{3}^{2}, y_{2} y_{6}=y_{3} y_{5}$ \\
\hline 9 & $y_{2} y_{7}=t_{3} y_{6}=0$ \\
\hline 10 & $t_{3} y_{7}=0, y_{2} y_{4}^{2}+y_{3}^{2} y_{4}+y_{3} y_{7}=0, y_{2} y_{4}^{2}+y_{2}^{2} y_{6}+y_{5}^{2}=0$ \\
\hline 11 & $y_{3} y_{4}^{2}+y_{3}^{2} y_{5}+y_{4} y_{7}+y_{5} y_{6}=0, y_{2} y_{3} y_{6}+y_{2} y_{4} y_{5}=0$ \\
\hline 12 & $y_{5} y_{7}=0, y_{2}^{2} y_{4}^{2}+y_{3}^{4}+y_{3}^{2} y_{6}+y_{4}^{3}+y_{6}^{2}=0$ \\
\hline 13 & $y_{3} y_{4} y_{6}+y_{4}^{2} y_{5}+y_{6} y_{7}=0$ \\
\hline 14 & $y_{2} y_{4}^{3}+y_{3}^{2} y_{4}^{2}+y_{7}^{2}=0$ \\
\hline
\end{tabular}

except for the product $\sigma_{1} n_{1}=\sigma_{2}$. The non-trivial Bocksteins are $S q^{1}\left(n_{1}\right)=n_{1}^{2}$, $S q^{1}\left(m_{2}\right)=n_{1} m_{2}+n_{3}$, and $S q^{1}\left(m_{3}\right)=m_{2}^{2}$.

We apply the Bockstein spectral sequence and identify generators $y_{2} \leftrightarrow n_{1}^{2}=$ $S q^{1}\left(n_{1}\right) ; y_{3} \leftrightarrow n_{1} m_{2}+n_{3}=S q^{1}\left(m_{2}\right) ; y_{4} \leftrightarrow m_{2}^{2}=S q^{1}\left(m_{3}\right) ; y_{5} \leftrightarrow n_{1}^{2} m_{3}+$ $n_{1} m_{2}^{2}=S q^{1}\left(n_{1} m_{3}\right) ; y_{6} \leftrightarrow n_{1} m_{2} m_{3}+n_{3} m_{3}+m_{2}^{3}=S q^{1}\left(m_{2} m_{3}\right) ;$ and $y_{7} \leftrightarrow m_{2}^{2} n_{3}=$ $S q^{1}\left(m_{3} n_{3}\right)$. Table 3 contains the relations by degree.

It remains to determine what happens to the classes $s_{1}$ and $s_{2}$, and whether there are classes in the integral cohomology with order larger than 2. We use Flöge's presentation for the group: $\Gamma_{2}=\left\langle A, V, S, M, U ; A^{2}=S^{3}=(A M)^{2}=M^{2}=V^{3}=\right.$ 1, $\left.A M=S V^{2}, U^{-1} A U=M, U^{-1} S U=V\right\rangle$ [6]. Set $G=\left\langle A, V, S, M ; A^{2}=M^{2}=\right.$ $\left.(A M)^{2}=S^{3}=V^{3}=1, A M=S V^{2}\right\rangle$ and consider the subgroups

$$
\begin{aligned}
G_{1} & =\left\langle A, M ; A^{2}=M^{2}=(A M)^{2}=1\right\rangle \cong \mathbf{D}_{2}, \\
G_{2} & =\left\langle S, V ; S^{3}=V^{3}=\left(S V^{2}\right)^{2}=1\right\rangle \cong \mathbf{A}_{4}, \\
H & =\left\langle A M=S V^{2},(A M)^{2}=1\right\rangle \cong \mathbb{Z} / 2 .
\end{aligned}
$$

Let $G$ be the amalgamated product $G_{1} *_{H} G_{2} \cong \mathbf{D}_{2 * \mathbb{Z} / 2} \mathbf{A}_{4}$. Then $\Gamma_{2}=G *_{P S L_{2}(\mathbb{Z})}$, where $P S L_{2}(\mathbb{Z})=\langle A, S\rangle$ and the twisting is induced by the group element $U$.

We have already calculated $H^{*}(G ; \mathbb{Z})_{(2)}$ in Lemma 3.8, but in order to keep track of the classes more carefully, we look at this example again from the point of view of group structure. The amalgamated subgroup in $G$ is $\langle A M\rangle$, so let the two classes $u_{2}, v_{2} \in H^{2}\left(\mathbf{D}_{2} ; \mathbb{Z}\right)$ correspond to the subgroups $\langle A M\rangle$ and $\langle A\rangle$. Note that $\alpha$ sends $u_{2}$ to the generator of $H^{2}(\mathbb{Z} / 2 ; \mathbb{Z})$ but sends $v_{2}$ to 0 . The map $\alpha$ in sequence (3.1) is a surjection by Lemma 3.5 and Remark 3.6. so the long exact sequence splits into short exact sequences. It follows that in $H^{*}(G ; \mathbb{Z})_{(2)}$ there is a single class in degree 2 and two classes in degree 3 , all of order 2.

Next consider the HNN extension that generates $\Gamma_{2}$, where the group element $U$ twists $\langle A\rangle$ to $\langle M\rangle$. As $P S L_{2}(\mathbb{Z}) \cong \mathbb{Z} / 2 * \mathbb{Z} / 3$, let $w_{2}$ be the generator of $H^{2}\left(P S L_{2}(\mathbb{Z}) ; \mathbb{Z}\right)_{(2)}$ corresponding to the subgroup $\langle A\rangle$. Then $\operatorname{res}_{P S L_{2}(\mathbb{Z})}^{G}\left(v_{2}\right)=$ $\theta^{*}\left(v_{2}\right)=w_{2}$, so $\alpha\left(v_{2}\right)=0$. We can fit this information into sequence (2.11) for an HNN extension:

$$
0 \rightarrow H^{2}\left(\Gamma_{2} ; \mathbb{Z}\right)_{(2)} \stackrel{\rho}{\longrightarrow} \mathbb{Z} / 2 \stackrel{\alpha}{\longrightarrow} \mathbb{Z} / 2 \stackrel{\delta}{\longrightarrow} H^{3}\left(\Gamma_{2} ; \mathbb{Z}\right)_{(2)} \stackrel{\rho}{\longrightarrow} \mathbb{Z} / 2 \oplus \mathbb{Z} / 2 \rightarrow 0 .
$$


The map $\alpha$ is 0 , so $\rho$ is an isomorphism. We abuse notation and denote the class in $H^{2}\left(\Gamma_{2} ; \mathbb{Z}\right)_{(2)}$ by $v_{2}$ as well. (Notice that $\operatorname{res}_{P S L_{2}(\mathbb{Z})}^{\Gamma_{2}}\left(v_{2}\right)=w_{2}$, as both classes are dual to the group element $A$.) Since the Bockstein spectral sequence guarantees the existence of a single class of order 2 in $H^{3}\left(\Gamma_{2} ; \mathbb{Z}\right)_{(2)}$, there must be another class in degree 3 of order 4 , denoted by $t_{3}$. Also, there is a torsion-free HNN class in degree $1, \sigma_{1}$. This class has a trivial product with $t_{3}$ by Lemma 2.4. However, by Lemma 2.3. $\sigma_{1} v_{2}=\delta(1) \cup v_{2}=\delta\left(1 \cup \operatorname{res}_{P S L_{2}(\mathbb{Z})}^{\Gamma_{2}}\left(v_{2}\right)\right)=\delta\left(w_{2}\right)=2 t_{3}$. As $\alpha$ is a surjection in degrees 3 and higher, the same lemma implies that the rest of the products with $\sigma_{1}$ are trivial. That $\alpha$ is surjective also implies that $t_{3}$ is the only 2 -torsion class of order greater than 2; using an argument similar to that in Lemma 3.9 implies that all products with $t_{3}$ are trivial.

To determine $H^{*}\left(\Gamma_{2} ; \mathbb{Z}\right)_{(3)}$, we have that $H^{*}(G ; \mathbb{Z})_{(3)} \cong H^{*}(\mathbb{Z} / 3 ; \mathbb{Z})$, so all that remains is to determine the effect of the final HNN extension. The group element $U$ sends $S$ to $V$, which are both elements of degree 3 in $\mathbf{A}_{4}$. Therefore, both the injection and twisting maps are isomorphisms, which implies that $\alpha$ is the zero map. We get short exact sequences

$$
0 \rightarrow H^{*}\left(P S L_{2}(\mathbb{Z}) ; \mathbb{Z}\right)_{(3)} \stackrel{\delta}{\longrightarrow} H^{*+1}\left(\Gamma_{2} ; \mathbb{Z}\right)_{(3)} \stackrel{\rho}{\longrightarrow} H^{*+1}(\mathbb{Z} / 3 ; \mathbb{Z}) \stackrel{\alpha}{\longrightarrow} 0 .
$$

These yield classes $x_{2}^{n} \in H^{2 n}\left(\Gamma_{2} ; \mathbb{Z}\right)_{(3)}$ and $\delta\left(w_{2}^{n}\right) \in H^{2 n+1}\left(\Gamma_{2} ; \mathbb{Z}\right)_{(3)}$, where $w_{2}$ is the generator of $H^{2}\left(P S L_{2}(\mathbb{Z}) ; \mathbb{Z}\right)_{(3)}$. All classes have order 3 . There is also a torsion-free HNN class, $\delta(1) \in H^{1}\left(\Gamma_{2} ; \mathbb{Z}\right)_{(3)}$, which is the class $\sigma_{1}$ we identified before. We claim that products with this class generate the odd-dimensional cohomology. Note that $\operatorname{res}_{P S L_{2}(\mathbb{Z})}^{\Gamma_{2}}\left(x_{2}^{n}\right)=w_{2}^{n}$. Thus, $\delta\left(w_{2}^{n}\right)=\delta\left(w_{2}^{n} \cup 1\right)=$ $\delta\left(\operatorname{res}_{P S L_{2}(\mathbb{Z})}^{\Gamma_{2}}\left(x_{2}^{n}\right) \cup 1\right)=x_{2}^{n} \cup \delta(1)=x_{2}^{n} \cup \sigma_{1}$.

Theorem 4.2. $H^{*}\left(\Gamma_{6} ; \mathbb{Z}\right)_{(2)} \cong \mathbb{Z}\left[y_{3}, y_{4}, y_{6}, z_{6}, y_{7}, y_{9}\right] /\langle R\rangle \widetilde{\oplus} \mathbb{Z}\left[y_{2}\right]\left(\tau_{1}\right) \widetilde{\oplus} \mathbb{Z}\left(t_{3}\right) \widetilde{\oplus}$ $\mathbb{Z}\left(\sigma_{1}\right) \widetilde{\oplus} \mathbb{Z}\left(\sigma_{2}\right)$. The class $t_{3}$ has order 4 ; all other torsion generators have order 2. The relations $\langle R\rangle$ are identical to those for $H^{*}\left(\mathbf{A}_{4} *_{\mathbb{Z} / 2} \mathbf{A}_{4} ; \mathbb{Z}\right)_{(2)}$, and are given in Table 2, At the prime $3, H^{*}\left(\Gamma_{6} ; \mathbb{Z}\right)_{(3)} \cong \mathbb{Z}\left[x_{2}\right]\left(\tau_{1}\right) \widetilde{\oplus} \mathbb{Z}\left(\sigma_{1}\right) \widetilde{\oplus} \mathbb{Z}\left(\sigma_{2}\right)$ where $x_{2}$ has order 3 .

Proof. $H^{*}\left(\Gamma_{6} ; \mathbb{F}_{2}\right) \cong \mathbb{F}_{2}\left[u_{2}, v_{3}, \bar{v}_{3}, w_{3}\right] \widetilde{\oplus} \mathbb{F}_{2}\left[y_{1}\right]\left(s_{1}\right) \widetilde{\oplus} \mathbb{F}_{2}\left(t_{1}\right) \widetilde{\oplus} \mathbb{F}_{2}\left(s_{2}\right) \widetilde{\oplus} \mathbb{F}_{2}\left(t_{2}\right)$ with relations $u_{2}^{3}+w_{3}^{2}+v_{3}^{2}+\bar{v}_{3}^{2}+w_{3}\left(v_{3}+\bar{v}_{3}\right)=0, v_{3} \bar{v}_{3}=0$. The Bocksteins are $S q^{1}\left(y_{1}\right)=y_{1}^{2}$, $S q^{1}\left(u_{2}\right)=v_{3}+\bar{v}_{3}$ and $S q^{1}\left(w_{3}\right)=u_{2}^{2}[2]$.

As $\Gamma_{6}$ and $\mathbf{A}_{4} *_{\mathbb{Z} / 2} \mathbf{A}_{4}$ have almost identical mod-2 cohomology, it is no surprise that most of their generators and relations in the integral cohomology are identical too; we refer the reader to Lemma 3.9 for the definitions of $y_{3}, y_{4}, y_{6}, z_{6}, z_{7}$, and $y_{9}$, and their relations in Table 2. However, $\Gamma_{6}$ has a new class, $y_{2}$, that corresponds to $y_{1}^{2}=S q^{1}\left(y_{1}\right)$. We switch techniques and directly calculate the cohomology of $\Gamma_{6}$ using its group structure to find relations with $y_{2}$.

Flöge's presentation for $\Gamma_{6}$ is $\left\langle A, B, M, R, S, U, W ; A^{2}=B^{2}=M^{2}=R^{3}=\right.$ $S^{3}=(B R)^{3}=(B S)^{3}=1, A S=M R, U^{-1} A U=M, U^{-1} S U=R, W^{-1} M W=$ $\left.A, W^{-1} R B W=S B\right\rangle[6$. Let $G$ be the subgroup generated by the group elements $A, B, M, R, S$ and their relations. The following subgroups of $G$,

$$
\begin{aligned}
G_{1} & =\left\langle S, A, B ; A^{2}=S^{3}=B^{2}=(B S)^{3}=1\right\rangle \cong \mathbb{Z} / 2 * \mathbf{A}_{4}, \\
G_{2} & =\left\langle R, B, M ; M^{2}=R^{3}=B^{2}=(B R)^{3}=1\right\rangle \cong \mathbb{Z} / 2 * \mathbf{A}_{4}, \\
H & =\left\langle B, A S=M R ; B^{2}=1\right\rangle \cong \mathbb{Z} / 2 * \mathbb{Z},
\end{aligned}
$$


combine to form $G \cong G_{1} *_{H} G_{2} \cong\left(\mathbb{Z} / 2 * \mathbf{A}_{4}\right) *(\mathbb{Z} / 2 * \mathbb{Z})\left(\mathbb{Z} / 2 * \mathbf{A}_{4}\right)$. The associated subgroups of the HNN extensions, $\langle A, S\rangle$ and $\langle M, R B\rangle$, are both isomorphic to $P S L_{2}(\mathbb{Z})$. We add the group element $U$ to get the HNN extension $G_{3}=G *_{P S L_{2}(\mathbb{Z})}$. Finally, we add $W$ to get $\Gamma_{6}=G_{3} *_{P S L_{2}(\mathbb{Z})}$. Thus, the cohomology of $\Gamma_{6}$ can be calculated in three steps.

We use sequence (2.1) to find $H^{*}(G ; \mathbb{Z})_{(2)}$.

$$
\begin{aligned}
& \ldots \stackrel{\delta}{\longrightarrow} H^{*}(G ; \mathbb{Z})_{(2)} \stackrel{\rho}{\longrightarrow} H^{*}\left(\mathbb{Z} / 2 * \mathbf{A}_{4} ; \mathbb{Z}\right)_{(2)} \oplus H^{*}\left(\mathbb{Z} / 2 * \mathbf{A}_{4} ; \mathbb{Z}\right)_{(2)} \\
& \quad \stackrel{\alpha}{\longrightarrow} H^{*}(\mathbb{Z} / 2 * \mathbb{Z} ; \mathbb{Z}) \stackrel{\delta}{\longrightarrow} \ldots
\end{aligned}
$$

From the group presentation, we see that the amalgamated copy of $\mathbb{Z} / 2$ injects into both copies of $\mathbf{A}_{4}$, forming $\mathbf{A}_{4} *_{\mathbb{Z} / 2} \mathbf{A}_{4}$ as a subgroup. The only torsion-free class in the sequence, in $H^{1}(\mathbb{Z} ; \mathbb{Z})$, is not hit by $\alpha$. Its image under $\delta$ yields a torsion-free class in degree 2 that we denote by $\sigma_{2}$. Since this class and $2 t_{3}$ are the only ones in the image of $\delta$, they have trivial products with the rest of the ring elements by Lemma 2.3 and with each other by Lemma 2.4. The other copies of $\mathbb{Z} / 2$, corresponding to the group elements $A$ and $M$, go to 0 under $\alpha$. Thus, they appear as two copies of $H^{*}(\mathbb{Z} / 2 ; \mathbb{Z})$ in $H^{*}(G ; \mathbb{Z})_{(2)}$, which is isomorphic to the reduced direct sum $H^{*}\left(\mathbf{A}_{4} * \mathbb{Z} / 2 \mathbf{A}_{4} ; \mathbb{Z}\right)_{(2)} \widetilde{\oplus} H^{*}(\mathbb{Z} / 2 ; \mathbb{Z}) \widetilde{\oplus} H^{*}(\mathbb{Z} / 2 ; \mathbb{Z}) \widetilde{\oplus} \mathbb{Z}\left(\sigma_{2}\right)$. Let $v_{2}$ and $w_{2}$, dual to $\langle A\rangle$ and $\langle M\rangle$, generate the two copies of $H^{2}(\mathbb{Z} / 2 ; \mathbb{Z})$.

For the first HNN extension, the element $U$ twists $A$ to $M$, with $\langle A\rangle \subseteq P S L_{2}(\mathbb{Z})$, the associated subgroup of the extension. By sequence (2.1), we get the sequence

$$
\ldots \stackrel{\delta}{\longrightarrow} H^{*}\left(G_{3} ; \mathbb{Z}\right)_{(2)} \stackrel{\rho}{\longrightarrow} H^{*}(G ; \mathbb{Z})_{(2)} \stackrel{\alpha}{\longrightarrow} H^{*}\left(P S L_{2}(\mathbb{Z}) ; \mathbb{Z}\right)_{(2)} \stackrel{\delta}{\longrightarrow} \ldots
$$

Now as $\alpha$ is a surjection in positive degrees, the long exact sequence breaks into short exact sequences. In $H^{*}(G ; \mathbb{Z})_{(2)}$, the sum of the two generators $v_{2}+w_{2}$ is in the kernel of $\alpha$ because of the twisting; let $y_{2}$ be the inverse image under $\rho$ of this new generator. There is also an HNN class, $\sigma_{1}$, that has trivial products as it is the only class that arises from the image of $\delta$. Thus, $H^{*}\left(G_{3} ; \mathbb{Z}\right)_{(2)} \cong$ $H^{*}\left(\mathbf{A}_{4} *_{\mathbb{Z} / 2} \mathbf{A}_{4} ; \mathbb{Z}\right)_{(2)} \widetilde{\oplus} H^{*}(\mathbb{Z} / 2 ; \mathbb{Z}) \widetilde{\oplus} \mathbb{Z}\left(\sigma_{1}\right) \widetilde{\oplus} \mathbb{Z}\left(\sigma_{2}\right)$.

The final HNN extension yields $H^{*}\left(\Gamma_{6} ; \mathbb{Z}\right)_{(2)}$. Here the twisting sends $M$ back to $A$. In terms of the maps induced by inclusion of twisting, $\operatorname{res}_{P S L_{2}(\mathbb{Z})}^{G_{3}}\left(w_{2}\right)=\theta^{*}\left(v_{2}\right)$, so $\alpha\left(y_{2}\right)=0$. We abuse notation and let $y_{2}$ also denote its inverse image in $H^{*}\left(\Gamma_{6} ; \mathbb{Z}\right)_{(2)}$ that arises from the kernel of $\alpha$. Sequence (2.1) breaks into short exact sequences that include a degree shift:

$$
0 \stackrel{\alpha}{\longrightarrow} H^{*}\left(P S L_{2}(\mathbb{Z}) ; \mathbb{Z}\right)_{(2)} \stackrel{\delta}{\longrightarrow} H^{*+1}\left(\Gamma_{6} ; \mathbb{Z}\right)_{(2)} \stackrel{\rho}{\longrightarrow} H^{*+1}\left(G_{3} ; \mathbb{Z}\right)_{(2)} \stackrel{\alpha}{\longrightarrow} 0 .
$$

New classes in $H^{*}(\Gamma ; \mathbb{Z})_{(2)}$ include the HNN class $\tau_{1}=\delta(1)$ and $\delta\left(u_{2}^{j}\right)$ in all odd degrees, where $u_{2} \in H^{2}\left(P S L_{2}(\mathbb{Z}) ; \mathbb{Z}\right)_{(2)}$ corresponds to the copy of $\mathbb{Z} / 2$ generated by the group element $M$. We use Lemma 2.3 to show that the new classes in odd degree are actually products of other classes. Let $i$ denote the subgroup inclusion $P S L_{2}(\mathbb{Z}) \hookrightarrow \Gamma_{6}$. By construction, $\operatorname{res}_{P S L_{2}(\mathbb{Z})}^{\Gamma_{6}}\left(y_{2}\right)=u_{2}$. Therefore, $\delta\left(u_{2}^{k}\right)=\delta\left(\operatorname{res}_{P S L_{2}(\mathbb{Z})}^{\Gamma_{6}}\left(y_{2}^{k}\right) \cup 1\right)=y_{2}^{k} \cup \delta(1)=y_{2}^{k} \tau_{1}$.

The 3-primary cohomology calculation is similar. First,

$$
H^{*}(G ; \mathbb{Z})_{(3)} \cong H^{*}(\mathbb{Z} / 3 ; \mathbb{Z}) \widetilde{\oplus} H^{*}(\mathbb{Z} / 3 ; \mathbb{Z}) \widetilde{\oplus} \mathbb{Z}\left(\sigma_{2}\right) .
$$

The torsion-free class, $\sigma_{2}$, is the image of $H^{1}(\mathbb{Z} ; \mathbb{Z})$ under $\delta$. The first HNN extension has the effect of identifying the two polynomial classes as it twists one copy of $\mathbb{Z} / 3$ 
to the other. Thus, $H^{*}\left(G_{3} ; \mathbb{Z}\right)_{(3)} \cong H^{*}(\mathbb{Z} / 3 ; \mathbb{Z}) \widetilde{\oplus} \mathbb{Z}\left(\sigma_{1}\right) \widetilde{\oplus} \mathbb{Z}\left(\sigma_{2}\right)$, where $\sigma_{1}$ is the torsion-free HNN class.

For the final HNN extension, $W$ twists the second copy of $\mathbb{Z} / 3$ back to the original one; thus $\alpha$ is 0 . The long exact sequence splits as in the case of $\Gamma_{2}$, with the same results: we get a new exterior $\mathrm{HNN}$ class $\tau_{1}$, that multiplies non-trivially with a new polynomial class in degree 2 .

5. The Bianchi groups $\Gamma_{1}, \Gamma_{3}, \Gamma_{5}, \Gamma_{7}, \Gamma_{10}$, And $\Gamma_{11}$

Theorem 5.1. $H^{*}\left(\Gamma_{1} ; \mathbb{Z}\right)_{(2)} \cong H^{*}\left(\mathbf{A}_{4} ; \mathbb{Z}\right)_{(2)} \widetilde{\oplus} H^{*}\left(\mathbf{D}_{2} ; \mathbb{Z}\right)_{(2)} . \quad H^{*}\left(\Gamma_{1} ; \mathbb{Z}\right)_{(3)} \cong$ $\mathbb{Z}\left[x_{4}\right]\left(x_{3}\right)$ where $x_{3}$ and $x_{4}$ have order 3 .

Proof. Fine shows that $\Gamma_{1}$ has the group presentation $\left\langle A, B, C, D ; A^{3}=B^{2}=C^{3}=\right.$ $\left.D^{2}=(A C)^{2}=(A D)^{2}=(B D)^{2}=(B C)^{2}=1\right\rangle[5$. We break this into subgroups:

$$
\begin{aligned}
& G_{11}=\left\langle A, C ; A^{3}=C^{3}=(A C)^{2}=1\right\rangle \cong \mathbf{A}_{4}, \\
& G_{12}=\left\langle A, D ; A^{3}=D^{2}=(A D)^{2}=1\right\rangle \cong \mathbf{S}_{3}, \\
& G_{21}=\left\langle B, C ; B^{2}=C^{3}=(B C)^{2}=1\right\rangle \cong \mathbf{S}_{3}, \\
& G_{22}=\left\langle B, D ; B^{2}=D^{2}=(B D)^{2}=1\right\rangle \cong \mathbf{D}_{2} .
\end{aligned}
$$

Set $G_{1}=G_{11} *\langle A\rangle G_{12}$ and $G_{2}=G_{21} *\langle B\rangle G_{22}$. Then $\Gamma_{1}$ is an amalgamated product $G_{1} *_{P S L_{2}(\mathbb{Z})} G_{2}$, where $P S L_{2}(\mathbb{Z}) \cong\langle C, D\rangle$.

Using sequence (2.1) for an amalgamated product, one easily shows that

$$
H^{*}\left(G_{1} ; \mathbb{Z}\right)_{(2)} \cong H^{*}\left(\mathbf{A}_{4} ; \mathbb{Z}\right)_{(2)},
$$

as $\alpha$ maps $H^{*}\left(\mathbf{S}_{3} ; \mathbb{Z}\right)_{(2)}$ isomorphically onto $H^{*}(\mathbb{Z} / 2 ; \mathbb{Z})$. On the other hand, $H^{*}\left(G_{2} ; \mathbb{Z}\right)_{(2)} \cong H^{*}\left(\mathbf{D}_{2} ; \mathbb{Z}\right) \widetilde{\oplus} H^{*}\left(\mathbf{S}_{3} ; \mathbb{Z}\right)_{(2)}$. The 2-primary result follows by noting that the copy of $H^{*}\left(\mathbf{S}_{3} ; \mathbb{Z}\right)_{(2)}$ maps isomorphically onto $H^{*}\left(P S L_{2}(\mathbb{Z}) ; \mathbb{Z}\right)_{(2)}$ in the final amalgamated product.

For the 3-primary component, the restriction maps for $\mathbf{A}_{4}$ and $\mathbf{S}_{3}$ to $\mathbb{Z} / 3$ (Lemmas 3.2 and 3.4 imply that both $H^{*}\left(G_{1} ; \mathbb{Z}\right)_{(3)}$ and $H^{*}\left(G_{2} ; \mathbb{Z}\right)_{(3)} \cong \mathbb{Z}\left[x_{4}\right]$ where $x_{4}$ has order 3. Using sequence (2.1) we get

$$
\begin{aligned}
\ldots \stackrel{\delta}{\longrightarrow} H^{*}\left(\Gamma_{1} ; \mathbb{Z}\right)_{(3)} \stackrel{\rho}{\longrightarrow} H^{*}\left(G_{1} ; \mathbb{Z}\right)_{(3)} \oplus H^{*}\left(G_{2} ; \mathbb{Z}\right)_{(3)} \stackrel{\alpha}{\longrightarrow} H^{*-1}\left(P S L_{2}(\mathbb{Z}) ; \mathbb{Z}\right)_{(3)} \\
\stackrel{\delta}{\longrightarrow} H^{*}\left(\Gamma_{1} ; \mathbb{Z}\right)_{(3)} \stackrel{\rho}{\longrightarrow} H^{*}\left(G_{1} ; \mathbb{Z}\right)_{(3)} \oplus H^{2}\left(G_{2} ; \mathbb{Z}\right)_{(3)} \stackrel{\alpha}{\longrightarrow} \ldots
\end{aligned}
$$

Both classes in degree 4 map onto the square of the generator

$$
w_{2} \in H^{2}\left(P S L_{2}(\mathbb{Z}) ; \mathbb{Z}\right)_{(3)} .
$$

Therefore, we get classes $x_{4}^{n} \in H^{4 n}\left(\Gamma_{1} ; \mathbb{Z}\right)_{(3)}$ from the kernel of $\alpha$ and classes $\delta\left(w_{2}^{4 n+2}\right) \in H^{4 n+3}\left(\Gamma_{1} ; \mathbb{Z}\right)_{(3)}$. These latter classes are exterior by degree considerations. Note that $\operatorname{res}_{P S L_{2}(\mathbb{Z})}^{\Gamma_{1}}\left(x_{4}^{n}\right)=w_{2}^{2 n}$. Thus,

$$
\delta\left(w_{2}^{2 n+1}\right)=\delta\left(r e s_{P S L_{2}(\mathbb{Z})}^{\Gamma_{1}}\left(x_{4}^{n}\right) \cup w_{2}\right)=x_{4}^{n} \cup \delta\left(w_{2}\right)=x_{4}^{n} x_{3}
$$

by Lemma 2.3 ,

Theorem 5.2. $H^{*}\left(\Gamma_{3} ; \mathbb{Z}\right)_{(2)} \cong H^{*}\left(\mathbf{A}_{4} *_{\mathbb{Z} / 2} \mathbf{A}_{4} ; \mathbb{Z}\right)_{(2)} . H^{*}\left(\Gamma_{3} ; \mathbb{Z}\right)_{(3)} \cong \mathbb{Z}\left[x_{2}\right] \widetilde{\oplus} \mathbb{Z}\left[x_{4}\right]$, where both generators have order 3 . 
Proof. From calculations in [2], $H^{*}\left(\Gamma_{3} ; \mathbb{F}_{2}\right) \cong \mathbb{F}_{2}\left[u_{2}, v_{3}, \bar{v}_{3}, w_{3}\right] \widetilde{\oplus}_{2}\left(s_{2}\right)$ with relations $u_{2}^{3}+w_{3}^{2}+v_{3}^{2}+\bar{v}_{3}^{2}+w_{3}\left(v_{3}+\bar{v}_{3}\right)=0, v_{3} \bar{v}_{3}=0$. This is identical to $H^{*}\left(\mathbf{A}_{4} * \mathbb{Z} / 2 \mathbf{A}_{4} ; \mathbb{F}_{2}\right)$ given in Lemma 3.9 .

The 3-primary calculations are more involved. We use the fact that $\Gamma_{3}$ acts on a retract known as the "Mendoza Complex" [10] with fundamental domain and isotropy groups [13] given in Figure 1.

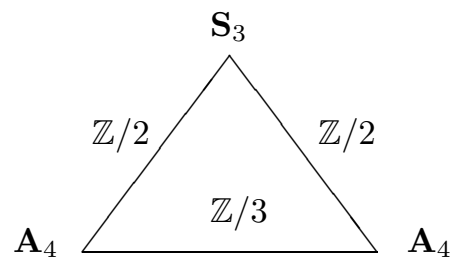

FiguRE 1. Fundamental domain for $\Gamma_{3}$

One now uses the equivariant spectral sequence (4], VII.7) to show that

$$
H^{*}\left(\Gamma_{3} ; \mathbb{Z}\right)_{(3)} \cong H^{*}\left(\mathbf{A}_{4} ; \mathbb{Z}\right)_{(3)} \widetilde{\oplus} H^{*}\left(\mathbf{S}_{3} ; \mathbb{Z}\right)_{(3)} .
$$

The details are identical to, but easier than, those presented in [2]. We refer the interested reader there.

Theorem 5.3. $H^{*}\left(\Gamma_{5} ; \mathbb{Z}\right)_{(2)} \cong \mathbb{Z}\left[y_{2}, z_{2}, y_{3}, y_{5}, z_{5}, y_{6}\right] /\langle R\rangle \widetilde{\oplus} \mathbb{Z}\left(t_{3}\right) \widetilde{\oplus} \mathbb{Z}\left(\sigma_{1}\right) \widetilde{\oplus} \mathbb{Z}\left(\tau_{1}\right)$ $\widetilde{\oplus} \mathbb{Z}\left(\sigma_{2}\right)$. The class $t_{3}$ has order 4 ; all other torsion generators have order two. The set of relations, $\langle R\rangle$, is given in Table $4 . H^{*}\left(\Gamma_{5} ; \mathbb{Z}\right)_{(3)} \cong \mathbb{Z}\left[x_{2}\right]\left(\tau_{1}\right) \widetilde{\oplus} \mathbb{Z}\left(\sigma_{1}\right) \widetilde{\oplus} \mathbb{Z}\left(\sigma_{2}\right)$, where $x_{2}$ has order 3 .

TABLE 4. Relations by degree for $H^{*}\left(\Gamma_{5} ; \mathbb{Z}\right)_{(2)}$

\begin{tabular}{|l|l|}
\hline Degree & Relation(s) \\
\hline 6 & $y_{2} z_{2}^{2}+y_{2}^{2} z_{2}+y_{3}^{2}=0$ \\
\hline 7 & $y_{2} z_{5}=z_{2} y_{5}$ \\
\hline 8 & $y_{2} y_{6}=y_{3} y_{5}, y_{3} z_{5}=z_{2} y_{6}$ \\
\hline 10 & $y_{2}^{2} y_{6}=y_{5}^{2}, z_{2}^{2} y_{6}=z_{5}^{2}$ \\
\hline 12 & $y_{2}^{2} z_{2} y_{6}+y_{2} z_{2}^{2} y_{6}+y_{6}^{2}=0$ \\
\hline
\end{tabular}

Proof. From [2, $H^{*}\left(\Gamma_{5} ; \mathbb{F}_{2}\right) \cong \mathbb{F}_{2}\left[l_{1}, m_{1}, m_{3}\right] \widetilde{\oplus} \mathbb{F}_{2}\left(s_{1}\right) \widetilde{\oplus} \mathbb{F}_{2}\left(s_{2}\right) \widetilde{\oplus} \mathbb{F}_{2}\left(t_{1}\right) \widetilde{\oplus} \mathbb{F}_{2}\left(t_{2}\right)$ with relation $m_{3}^{2}+l_{1}^{2} m_{1} m_{3}+l_{1} m_{1}^{2} m_{3}=0$. The Bocksteins are $S q^{1}\left(l_{1}\right)=l_{1}^{2}$ and $S q^{1}\left(m_{1}\right)=m_{1}^{2}$. Enumerating classes by degree, we find the six generators mentioned above: $y_{2} \leftrightarrow l_{1}^{2}=S q^{1}\left(l_{1}\right) ; z_{2} \leftrightarrow m_{1}^{2}=S q^{1}\left(m_{1}\right) ; y_{3} \leftrightarrow l_{1}^{2} m_{1}+l_{1} m_{1}^{2}=$ $S q^{1}\left(l_{1} m_{1}\right) ; y_{5} \leftrightarrow l_{1}^{2} m_{3}=S q^{1}\left(l_{1} m_{3}\right) ; z_{5} \leftrightarrow m_{1}^{2} m_{3}=S q^{1}\left(m_{1} m_{3}\right) ;$ and $y_{6} \leftrightarrow m_{3}^{2}=$ $S q^{1}\left(l_{1} m_{1} m_{3}\right)$. This last relation follows as $m_{3}^{2}=l_{1}^{2} m_{1} m_{3}+l_{1} m_{1}^{2} m_{3}$ in mod-2 cohomology. These six generators satisfy the relations in Table 4

We use the group structure to finish the calculations. Flöge's presentation for $\Gamma_{5}$ is $\left\langle A, B, M, R, S, U, W ; A^{2}=B^{2}=M^{2}=R^{3}=S^{3}=(A B)^{2}=(B M)^{2}=1, A S=\right.$ $\left.M R, U^{-1} A U=M, U^{-1} S U=R, W^{-1} M B W=A B, W^{-1} R W=S\right\rangle$ [6]. We write 
this as a double HNN extension, with base group $G$, generated by $\langle A, B, M, R, S\rangle$. Consider the subgroups

$$
\begin{aligned}
G_{1} & =\left\langle S, A, B ; S^{3}=A^{2}=B^{2}=(A B)^{2}=1\right\rangle \cong \mathbb{Z} / 3 * \mathbf{D}_{2} \\
G_{2} & =\left\langle R, B, M ; R^{3}=B^{2}=M^{2}=(M B)^{2}=1\right\rangle \cong \mathbb{Z} / 3 * \mathbf{D}_{2} \\
H & =\left\langle B, A S=M R ; B^{2}=1\right\rangle \cong \mathbb{Z} / 2 * \mathbb{Z} .
\end{aligned}
$$

Thus $G \cong G_{1} *_{H} G_{2} \cong\left(\mathbb{Z} / 3 * \mathbf{D}_{2}\right) *(\mathbb{Z} / 2 * \mathbb{Z})\left(\mathbf{D}_{2} * \mathbb{Z} / 3\right)$. Adding the group element $U$, set $G_{3}=G *_{P S L_{2}(\mathbb{Z})}$. Adding $W, \Gamma_{5}=G_{3} *_{P S L_{2}(\mathbb{Z})}$. Both associated subgroups, $\left\langle A, S ; A^{2}=S^{3}=1\right\rangle$ and $\left\langle M B, R ;(M B)^{2}=R^{3}=1\right\rangle$, are isomorphic to $P S L_{2}(\mathbb{Z})$.

Start with sequence (2.1) for an amalgamated product:

$$
\ldots \stackrel{\delta}{\longrightarrow} H^{*}(G ; \mathbb{Z})_{(2)} \stackrel{\rho}{\longrightarrow} H^{*}\left(\mathbf{D}_{2} ; \mathbb{Z}\right) \oplus H^{*}\left(\mathbf{D}_{2} ; \mathbb{Z}\right) \stackrel{\alpha}{\longrightarrow} H^{*}(\mathbb{Z} / 2 * \mathbb{Z} ; \mathbb{Z})_{(2)} \stackrel{\delta}{\longrightarrow} \ldots
$$

By Lemma 3.7 we see that $H^{*}(G ; \mathbb{Z})_{(2)} \cong H^{*}\left(\mathbf{D}_{2} *_{\mathbb{Z} / 2} \mathbf{D}_{2}\right) \widetilde{\oplus} \mathbb{Z}\left(\sigma_{2}\right) ; \sigma_{2}$ is the image under $\delta$ of the torsion-free class in $H^{1}(\mathbb{Z} / 2 * \mathbb{Z} ; \mathbb{Z})$. Explicitly, $H^{*}(G ; \mathbb{Z})_{(2)} \cong$ $\mathbb{Z}\left[x_{2}, y_{2}, z_{2}, y_{3}, z_{3}\right] /\left\langle R^{\prime}\right\rangle \widetilde{\oplus} \mathbb{F}_{2}\left(\sigma_{2}\right)$, where the relations $\left\langle R^{\prime}\right\rangle$ are as in Lemma 3.7 Assume that $y_{2}$ and $z_{2}$ are associated to $\langle A\rangle$ and $\langle M\rangle$, respectively.

Next we add $U$ (twisting $A$ to $M$ ) to get $G_{3}$. By sequence (2.1),

$$
\ldots \stackrel{\delta}{\longrightarrow} H^{*}\left(G_{3} ; \mathbb{Z}\right)_{(2)} \stackrel{\rho}{\longrightarrow} H^{*}(G ; \mathbb{Z})_{(2)} \stackrel{\alpha}{\longrightarrow} H^{*}\left(P S L_{2}(\mathbb{Z}) ; \mathbb{Z}\right)_{(2)} \stackrel{\delta}{\longrightarrow} \ldots
$$

Denote the HNN class by $\sigma_{1}$. The map $\alpha$ is surjective in positive even dimensions, and as $\operatorname{res}_{P S L_{2}(\mathbb{Z})}^{G}\left(y_{2}\right)=\theta^{*}\left(z_{2}\right), y_{2}+z_{2}$ is in the kernel of $\alpha . H^{2}\left(G_{3} ; \mathbb{Z}\right)_{(2)}$ contains two classes: $l_{2}=y_{2}+z_{2}$, and $m_{2}$, which is the preimage of $x_{2}$. Also, $\alpha$ is the zero map in degree 3 , so $H^{3}\left(G_{3} ; \mathbb{Z}\right)_{(2)}$ contains the two classes corresponding to those in $H^{3}(G ; \mathbb{Z})_{(2)}$. Finally, $H^{*}\left(G_{3} ; \mathbb{Z}\right)_{(2)}$ has a new class in degree $4\left(\right.$ as $\left.\alpha\left(x_{2} y_{2}\right)=0\right)$ that we denote by $z_{4}$.

The final calculation builds $\Gamma_{5}$ from $G_{3}$ by adding the group element $W$. This element twists a copy of $P S L_{2}(\mathbb{Z})$, sending $\langle M B\rangle$ to $\langle A B\rangle$. Denote by $w_{2}$ the generator of $H^{2}\left(P S L_{2}(\mathbb{Z}) ; \mathbb{Z}\right)_{(2)}$. In terms of cohomology, $\operatorname{res}_{P S L_{2}(\mathbb{Z})}^{G_{3}}\left(x_{2}\right)=\operatorname{res}_{P S L_{2}(\mathbb{Z})}^{G_{3}}\left(z_{2}\right)=$ $w_{2}$, and $\theta^{*}\left(x_{2}\right)=\theta^{*}\left(y_{2}\right)=w_{2}$. Therefore, $\alpha$ sends both $l_{2}$ and $m_{2}$ to zero, so $\delta\left(w_{2}\right)$ has a non-trivial image in $H^{3}\left(\Gamma_{5} ; \mathbb{Z}\right)_{(2)}$. In addition, $\alpha$ is the zero map in degree 3 . Thus, there is a short exact sequence

$$
0 \stackrel{\alpha}{\longrightarrow} \mathbb{Z} / 2 \stackrel{\delta}{\longrightarrow} H^{3}\left(\Gamma_{5} ; \mathbb{Z}\right)_{(2)} \stackrel{\rho}{\longrightarrow} \mathbb{Z} / 2 \oplus \mathbb{Z} / 2 \stackrel{\alpha}{\longrightarrow} 0 .
$$

The calculations from the Bockstein spectral sequence imply that there is only one torsion class in degree 3 of order 2 . We conclude that $H^{3}\left(\Gamma_{5}\right)$ consists of a class of order 2 , and another class of order 4 that we call $t_{3}$. We also get an HNN class, $\tau_{1}$. That $t_{3}$ has trivial products follows from similar reasoning as in the calculation of $H^{*}\left(\mathbf{A}_{4} *_{\mathbb{Z} / 2} \mathbf{A}_{4} ; \mathbb{Z}\right)_{(2)}$ in Lemma 3.9. That $\tau_{1}$ has trivial products follows as it is the only class in the image of $\delta$. To finish the argument, $\alpha$ is surjective in all other degrees. For example, it is straightforward to check that $\alpha\left(z_{4}\right) \neq 0$. Thus, the final long exact sequence breaks into short exact sequences, which implies that all other 2-torsion classes have order 2. These have already been accounted for by the Bockstein spectral sequence, so the calculation is finished.

$H^{*}\left(\Gamma_{5} ; \mathbb{Z}\right)_{(3)}$ is calculated in an identical manner as the case $\Gamma_{6}$.

Theorem 5.4. $H^{*}\left(\Gamma_{7} ; \mathbb{Z}\right)_{(2)} \cong \mathbb{Z}\left[x_{2}\right]\left(\sigma_{1}\right)$, where $x_{2}$ has order 2 . $H^{*}\left(\Gamma_{7} ; \mathbb{Z}\right)_{(3)} \cong$ $\mathbb{Z}\left[x_{4}\right]\left(x_{3}\right) \widetilde{\oplus} \mathbb{Z}\left(\sigma_{1}\right)$, where both torsion generators have order 3 . 
Proof. From [2], $H^{*}\left(\Gamma_{7} ; \mathbb{F}_{2}\right) \cong \mathbb{F}_{2}\left[x_{1}\right]\left(y_{1}\right)$. The Bockstein spectral sequence yields one torsion class in the integral cohomology, $x_{2} \leftrightarrow x_{1}^{2}=S q^{1}\left(x_{1}\right)$, that is polynomial. The integral class is harder to detect.

We use an HNN description due to Fine, $\Gamma_{7} \cong\left(\mathbf{S}_{3} *_{\mathbb{Z} / 2} \mathbf{S}_{3}\right) *_{P S L_{2}(\mathbb{Z})}([5$, Theorem 4.4.2). The twisting sends a $\mathbb{Z} / 2$ in the first copy of $\mathbf{S}_{3}$ to the second, and a $\mathbb{Z} / 3$ in the second copy of $\mathbf{S}_{3}$ back to the first. Note that $H^{*}\left(\mathbf{S}_{3} *_{\mathbb{Z} / 2} \mathbf{S}_{3} ; \mathbb{Z}\right)_{(2)} \cong$ $H^{*}(\mathbb{Z} / 2 ; \mathbb{Z})$. Further, at the level of cohomology, the two-dimensional generators of each copy of $H^{*}\left(\mathbf{S}_{3} ; \mathbb{Z}\right)_{(2)}$ are identified in the amalgamated product.

We use sequence (2.1) for an HNN extension:

$$
\ldots \stackrel{\delta}{\longrightarrow} H^{*}\left(\Gamma_{7} ; \mathbb{Z}\right)_{(2)} \stackrel{\rho}{\longrightarrow} H^{*}\left(\mathbf{S}_{3} *_{\mathbb{Z} / 2} \mathbf{S}_{3} ; \mathbb{Z}\right)_{(2)} \stackrel{\alpha}{\longrightarrow} H^{*}\left(P S L_{2}(\mathbb{Z}) ; \mathbb{Z}\right)_{(2)} \stackrel{\delta}{\longrightarrow} \ldots
$$

The restriction and twisting maps coincide, so $\alpha=0$. We denote the generator of $H^{2}\left(P S L_{2}(\mathbb{Z}) ; \mathbb{Z}\right)_{(2)}$ by $w_{2}$, and the generator in $H^{2}\left(\Gamma_{7} ; \mathbb{Z}\right)_{(2)}$ by $x_{2}$, and note that $\operatorname{res}_{P S L_{2}(\mathbb{Z})}^{\Gamma_{7}}\left(x_{2}\right)=w_{2}$. There is also the HNN class, $\sigma_{1}$. Substituting, the long exact sequence breaks into short exact sequences:

$$
0 \rightarrow H^{*}(\mathbb{Z} / 2 ; \mathbb{Z}) \stackrel{\delta}{\longrightarrow} H^{*+1}\left(\Gamma_{7} ; \mathbb{Z}\right)_{(2)} \stackrel{\rho}{\longrightarrow} H^{*+1}\left(\mathbf{S}_{3} *_{\mathbb{Z} / 2} \mathbf{S}_{3} ; \mathbb{Z}\right)_{(2)} \rightarrow 0 .
$$

The classes in the odd dimensions are the products $x_{2}^{n} \sigma_{1}$. This follows from Lemma 2.3. as $\delta\left(w_{2}^{n}\right)=\delta\left(\operatorname{res}_{P S L_{2}(\mathbb{Z})}^{\Gamma_{7}}\left(x_{2}^{n}\right) \cup 1\right)=x_{2}^{n} \cup \delta(1)=x_{2}^{n} \sigma_{1}$.

At the prime $3, H^{*}\left(\mathbf{S}_{3} *_{\mathbb{Z} / 2} \mathbf{S}_{3} ; \mathbb{Z}\right)_{(3)} \cong H^{*}(\mathbb{Z} / 3 ; \mathbb{Z}) \widetilde{\oplus} H^{*}(\mathbb{Z} / 3 ; \mathbb{Z})$. The rest of the argument is similar to the case $\Gamma_{1}$, with the exception of the HNN class. Notice that no class in $H^{*}\left(\Gamma_{7} ; \mathbb{Z}\right)_{(3)}$ restricts to the generator of $H^{2}\left(P S L_{2}(\mathbb{Z}) ; \mathbb{Z}\right)_{(3)}$, so all products with the HNN class are trivial.

Theorem 5.5. $H^{*}\left(\Gamma_{10} ; \mathbb{Z}\right)_{(2)} \cong \mathbb{Z}\left[y_{2}, z_{2}, y_{3}, y_{5}, z_{5}, y_{6}\right] /\langle R\rangle \widetilde{\oplus} \mathbb{Z}\left(t_{3}\right) \widetilde{\oplus} \mathbb{Z}\left(\sigma_{1}\right) \widetilde{\oplus} \mathbb{Z}\left(\tau_{1}\right) \widetilde{\oplus}$ $\mathbb{Z}\left(\eta_{1}\right) \widetilde{\oplus} \mathbb{Z}\left(\sigma_{2}\right) \widetilde{\oplus} \mathbb{Z}\left(\tau_{2}\right)$. The class $t_{3}$ has order 4 ; all other torsion generators have order 2 . The torsion generators satisfy the same relations as the torsion generators of $H^{*}\left(\Gamma_{5} ; \mathbb{Z}\right)_{(2)}$, given in Table 4. $H^{*}\left(\Gamma_{10} ; \mathbb{Z}\right)_{(3)} \cong \mathbb{Z}\left[x_{2}\right]\left(\eta_{1}\right) \widetilde{\oplus} \mathbb{Z}\left(\sigma_{1}\right) \widetilde{\oplus} \mathbb{Z}\left(\tau_{1}\right) \widetilde{\oplus}$ $\mathbb{Z}\left(\sigma_{2}\right) \widetilde{\oplus} \mathbb{Z}\left(\tau_{2}\right)$ where $x_{2}$ has order 3 .

Proof. The torsion parts of $H^{*}\left(\Gamma_{10} ; \mathbb{Z}\right)_{(2)}$ and $H^{*}\left(\Gamma_{5} ; \mathbb{Z}\right)_{(2)}$ are identical because the polynomial classes in $H^{*}\left(\Gamma_{10} ; \mathbb{F}_{2}\right)$ and $H^{*}\left(\Gamma_{5} ; \mathbb{F}_{2}\right)$ are identical. The only difference is that $H^{*}\left(\Gamma_{10} ; \mathbb{F}_{2}\right)$ contains three torsion-free exterior classes in each of degrees 1 and 2 that have trivial products with all other classes [2].

Flöge's presentation for $\Gamma_{10}$ is $\left\langle A, B, L, S, D, U, W ; A^{2}=B^{2}=L^{2}=S^{3}=\right.$ $\left.(A B)^{2}=(A L)^{2}=1\right\rangle$ with other relations involving $D, U$, and $W$ that we give in their respective extensions [6]. The base group $G_{0}$ has the presentation $\langle A, B, L, S$; $\left.A^{2}=B^{2}=L^{2}=S^{3}=(A B)^{2}=(A L)^{2}=1\right\rangle$ which we break into pieces:

$$
\begin{aligned}
G_{01} & =\left\langle A, B ; A^{2}=(A B)^{2}=B^{2}=1\right\rangle \cong \mathbf{D}_{2}, \\
G_{02} & =\left\langle A, L ; A^{2}=(A L)^{2}=L^{2}=1\right\rangle \cong \mathbf{D}_{2}, \\
G_{03} & =\langle S\rangle \cong \mathbb{Z} / 3, \\
H & =\langle A\rangle \cong \mathbb{Z} / 2 .
\end{aligned}
$$

With this decomposition, $G_{0} \cong\left(G_{01} *_{H} G_{02}\right) * G_{03} \cong\left(\mathbf{D}_{2} * \mathbb{Z} / 2 \mathbf{D}_{2}\right) * \mathbb{Z} / 3$. The first HNN extension, $G_{1}$, adds the element $D$. Its presentation is $\left\langle G_{0}, D ; D^{-1} A L S D\right.$ $\left.=S^{-1} A B\right\rangle$, with $\langle A L S\rangle \cong \mathbb{Z} . G_{2}$, the second HNN extension, adds the group element $U$. Explicitly, $G_{2}=\left\langle G_{1}, U ; U^{-1} D A B D^{-1} U=D^{-1} A L D, U^{-1} L D S^{-1} D^{-1} U\right.$ 
$\left.=B D^{-1} S^{-1} D\right\rangle$. The associated subgroup $\left\langle D A B D^{-1}, L D S^{-1} D^{-1}\right\rangle$ is isomorphic to $\mathbb{Z} / 2 * \mathbb{Z}$. The final HNN extension is

$$
\Gamma_{10}=\left\langle G_{2}, W ; W^{-1} B W=U^{-1} L U, W^{-1} D^{-1} S D W=U^{-1} D S D^{-1} U\right\rangle,
$$

where the associated subgroup $\left\langle B, D^{-1} S D\right\rangle$ is isomorphic to $P S L_{2}(\mathbb{Z})$.

At the prime $2, H^{*}\left(G_{0} ; \mathbb{Z}\right)_{(2)} \cong H^{*}\left(\mathbf{D}_{2} *_{\mathbb{Z} / 2} \mathbf{D}_{2} ; \mathbb{Z}\right)$. For the first HNN extension we use sequence (2.1):

$$
\ldots \stackrel{\delta}{\longrightarrow} H^{*}\left(G_{1} ; \mathbb{Z}\right)_{(2)} \stackrel{\rho}{\longrightarrow} H^{*}\left(G_{0} ; \mathbb{Z}\right)_{(2)} \stackrel{\alpha}{\longrightarrow} H^{*}(\mathbb{Z} ; \mathbb{Z}) \stackrel{\delta}{\longrightarrow} \ldots
$$

This implies that $H^{*}\left(G_{1} ; \mathbb{Z}\right)_{(2)} \cong H^{*}\left(G_{0} ; \mathbb{Z}\right)_{(2)}$ with two exceptions: there are new torsion-free classes in degrees 1 and 2 . All products with these classes vanish by Lemmas 2.3 and 2.4 .

The next two HNN extensions proceed in a similar manner as the case $\Gamma_{5}$. These calculations account for the class $t_{3}$, and each HNN extension generates an HNN class in degree 1 . The second HNN extension also generates a torsion-free class in degree two, the image of $\delta\left(H^{1}(\mathbb{Z} * \mathbb{Z} / 2 ; \mathbb{Z})\right)$. Therefore, $H^{*}\left(\Gamma_{10} ; \mathbb{Z}\right)_{(2)}$ has the same torsion classes as $H^{*}\left(\Gamma_{5} ; \mathbb{Z}\right)_{(2)}$, as well as three torsion-free classes in degree 1 and two in degree 2.

$H^{*}\left(\Gamma_{10} ; \mathbb{Z}\right)_{(3)}$ is also very similar to $H^{*}\left(\Gamma_{5} ; \mathbb{Z}\right)_{(3)}$. Note that $H^{*}\left(G_{0} ; \mathbb{Z}\right)_{(3)} \cong$ $H^{*}(\mathbb{Z} / 3 ; \mathbb{Z})$. These are the only torsion classes in $H^{*}\left(G_{1} ; \mathbb{Z}\right)_{(3)}$ and $H^{*}\left(G_{0} ; \mathbb{Z}\right)_{(3)}$, as these HNN extensions twist copies of $\mathbb{Z}$ and $\mathbb{Z} * \mathbb{Z} / 2$. The HNN extensions also give rise to two torsion-free classes in each of the degrees 1 and 2 , the classes $\sigma_{1}$, $\sigma_{2}, \tau_{1}$, and $\tau_{2}$.

It remains to determine the effect of the final HNN extension, where a copy of $P S L_{2}(\mathbb{Z})$ is twisted. The original copy of $\mathbb{Z} / 3$ was generated by the group element $S$. Although it is not twisted by $W$, a conjugate of it is, which induces an isomorphism on cohomology. Therefore, we see that both the restriction and the twisting maps are isomorphisms onto $H^{*}(\mathbb{Z} / 3 ; \mathbb{Z})$, which implies $\alpha=0$. The long exact sequence breaks into short exact sequences, as in the case of $\Gamma_{5}$. We get an HNN class, $\eta_{1}=\delta(1)$, and this class multiplies non-trivially with powers of the generator in $H^{2}\left(\Gamma_{10} ; \mathbb{Z}\right)_{(3)}$ that arises from $\alpha$ being the zero map.

Theorem 5.6. $H^{*}\left(\Gamma_{11} ; \mathbb{Z}\right)_{(2)} \cong H^{*}\left(\mathbf{A}_{4} *_{\mathbb{Z} / 2} \mathbf{A}_{4} ; \mathbb{Z}\right)_{(2)} \widetilde{\oplus} \mathbb{Z}\left(\sigma_{1}\right) ;$ the generators and relations for $H^{*}\left(\mathbf{A}_{4} *_{\mathbb{Z} / 2} \mathbf{A}_{4} ; \mathbb{Z}\right)_{(2)}$ can be found in Lemma 3.9. $H^{*}\left(\Gamma_{11} ; \mathbb{Z}\right)_{(3)} \cong$ $\mathbb{Z}\left[x_{2}\right]\left(\sigma_{1}\right)$, where $x_{2}$ has order 3 .

Proof. The Bockstein spectral sequence generates almost the entire integral ring structure from the mod-2 cohomology of the group, which is

$$
H^{*}\left(\Gamma_{11} ; \mathbb{F}_{2}\right) \cong \mathbb{F}_{2}\left[u_{2}, v_{3}, \bar{v}_{3}, w_{3}\right] \widetilde{\oplus} \mathbb{F}_{2}\left(s_{1}\right) \widetilde{\oplus} \mathbb{F}_{2}\left(s_{2}\right)
$$

with relations $u_{2}^{3}+w_{3}^{2}+v_{3}^{2}+\bar{v}_{3}^{2}+w_{3}\left(v_{3}+\bar{v}_{3}\right)=0, v_{3} \bar{v}_{3}=0$. With the exception of the class $s_{1}$, this is identical to $H^{*}\left(\mathbf{A}_{4} *_{\mathbb{Z} / 2} \mathbf{A}_{4} ; \mathbb{F}_{2}\right)$. We need to determine what happens to the class $s_{1}$ integrally.

The alternate method of calculation resolves this issue. Fine shows in [5, Theorem 4.4.2, that $\Gamma_{11}$ admits a description as an HNN extension,

$$
\Gamma_{11} \cong\left(\mathbf{A}_{4} *_{\mathbb{Z} / 3} \mathbf{A}_{4}\right) *_{P S L_{2}(\mathbb{Z})},
$$

where the twisting sends copies of $\mathbb{Z} / 2$ and $\mathbb{Z} / 3$ from one $\mathbf{A}_{4}$ to the other. Using sequence (2.1) we get

$$
\ldots \stackrel{\delta}{\longrightarrow} H^{*}\left(\Gamma_{11} ; \mathbb{Z}\right)_{(2)} \stackrel{\rho}{\longrightarrow} H^{*}\left(\mathbf{A}_{4} *_{\mathbb{Z} / 3} \mathbf{A}_{4} ; \mathbb{Z}\right)_{(2)} \stackrel{\alpha}{\longrightarrow} H^{*}\left(P S L_{2}(\mathbb{Z}) ; \mathbb{Z}\right)_{(2)} \stackrel{\delta}{\longrightarrow} \ldots,
$$


with $H^{*}\left(P S L_{2}(\mathbb{Z}) ; \mathbb{Z}\right)_{(2)} \cong H^{*}(\mathbb{Z} / 2 ; \mathbb{Z})_{(2)}$. From this sequence we see there is a torsion-free HNN class, $\sigma_{1}$. This is the only class to arise from the image of $\delta$, so all products with $\sigma_{1}$ vanish. Also, $H^{*}\left(\mathbf{A}_{4} * \mathbb{Z} / 3 \mathbf{A}_{4} ; \mathbb{Z}\right)_{(2)} \cong H^{*}\left(\mathbf{A}_{4} ; \mathbb{Z}\right)_{(2)} \widetilde{\oplus} H^{*}\left(\mathbf{A}_{4} ; \mathbb{Z}\right)_{(2)}$. The result follows.

For the 3 -primary cohomology, start with $H^{*}\left(\mathbf{A}_{4} *_{\mathbb{Z} / 3} \mathbf{A}_{4} ; \mathbb{Z}\right)_{(3)} \cong H^{*}(\mathbb{Z} / 3 ; \mathbb{Z})$. The twisting sends $\mathbb{Z} / 3$ in one copy of $\mathbf{A}_{4}$ to the other copy of $\mathbf{A}_{4}$; when one calculates the cohomology of the HNN extension, this implies that $\alpha$ is zero. We now proceed as in the case $\Gamma_{2}$.

Remark 5.7. Since the ring of integers $\mathcal{O}_{d}$ contains number theoretic information, one might hope that some of this information would be detected in the cohomology ring of the corresponding Bianchi group $\Gamma_{d}$. We note, however, that the torsion classes in $H^{*}\left(\Gamma_{5} ; \mathbb{Z}\right)$ and $H^{*}\left(\Gamma_{10} ; \mathbb{Z}\right)$ are identical - the difference is in the torsionfree classes! It is unknown at this point if there is a connection between $H^{*}\left(\Gamma_{d} ; \mathbb{Z}\right)$ and $\mathcal{O}_{d}$, but if there is, it is likely that many more examples will need to be calculated to find it.

\section{REFERENCES}

1. A. Adem and J. Milgram, Cohomology of Finite Groups, Springer-Verlag, 1994. MR:1317096 (96f:20082)

2. E. Berkove, On the Mod-2 Cohomology of the Bianchi Groups, Trans. of the AMS., 352, no. 10 (2000), 4585-4602. MR1675241 (2001b:11043)

3. W. Browder Torsion in H-spaces, Ann. of Math. (2) 74, 1961, 24-51. MR0124891 (23:A2201)

4. K. Brown, Cohomology of Groups, Springer Verlag, 1993. MR.1324339 (96a:20072)

5. B. Fine, Algebraic Theory of the Bianchi Groups, Marcel Dekker, Inc. (Monographs and textbooks in pure and applied mathematics; 129), 1989. MR.1010229 (90h:20002)

6. D. Flöge, Zur Struktur der PSL $L_{2}$ über einigen imaginär-quadratischen Zahlringen, Math. Zeitschrift 183 (1983), 255-279. MR0704107 (85c:11043)

7. F. Grunewald and J. Schwermer, Subgroups of Bianchi Groups and Arithmetic Quotients of Hyperbolic 3-Space, Trans. of the AMS (1) 335 (Jan. 1993), 47-78. MR1020042 (93c:11024)

8. G. Lewis, The Integral Cohomology Rings of Groups of Order $p^{3}$, Trans. of the AMS 132 (1968), 501-529. MR0223430 (36:6478)

9. J. McCleary, A User's Guide to Spectral Sequences, Second Edition, Cambridge University Press (Cambridge Studies in advance mathematics; 58), 2001. MR1793722 (2002c:55027)

10. E. Mendoza, Cohomology of $\mathrm{PGL}_{2}$ Over Imaginary Quadratic Integers, Bonner Math. Schriften, Vol. 128, 1980. MR0611515 (82g:22012)

11. K. Pearson, Integral Cohomology and Detection of $\omega$-Basic 2-Groups, Math. of Computation, Vol. 65213 (Jan. 1996), 291-306. MR1322893 (96d:20056)

12. J. Pakianathan and E. Yalçin On Nilpotent Ideals in the Cohomology Ring of a Finite Group, Topology 42 (2003), 1155-1183. MR1978052 (2004d:20062)

13. J. Schwermer and K. Vogtmann, The Integral Homology of $S L_{2}$ and $P S L_{2}$ of Euclidean Imaginary Quadratic Integers, Comment. Math. Helvetica 58 (1983), 573-598. MR0728453 (86d:11046)

14. J. P. Serre, Trees, Springer-Verlag, 1980. MR0607504 (82c:20083)

15. E. Spanier, Algebraic Topology, 1st reprinted version, Springer-Verlag, 1989. MR0666554 (83i:55001)

16. K. Vogtmann, Rational Cohomology of Bianchi Groups, Math. Ann. 272 (1985), 399-419. MR 0799670 (87a:22025)

Department of Mathematics, Lafayette College, Easton, Pennsylvania 18042

E-mail address: berkovee@lafayette.edu 